\title{
Impedance modeling and its application to the analysis of the collective effects
}

\author{
A. Blednykh॰, G. Bassi, and V. Smaluk \\ Brookhaven National Laboratory, Upton, New York 11973, USA \\ R. Lindberg \\ Argonne National Laboratory, Argonne, Illinois 60439, USA
}

(Received 4 May 2021; accepted 13 September 2021; published 12 October 2021)

\begin{abstract}
Impedance modeling for accelerator applications has improved over the years, largely as a result of advances in simulation capabilities. While this modeling has been successful in reproducing certain measurements, it is still a significant challenge to predict collective effects in real machines. In this paper, we review our approach to impedance modeling and the subsequent simulations of collective effects. We discuss the choice of the electrodynamics codes and the required computer power resources, modeling of the geometric, and resistive wall impedances, their comparison with analytical approaches, and their application for simulating of the collective effects with tracking and beam-induced heating.
\end{abstract}

DOI: 10.1103/PhysRevAccelBeams.24.104801

\section{INTRODUCTION}

In this paper, we would like to share our experience with impedance calculation, optimization, and its subsequent application to the analysis of collective effects. Our work is based on several years of research and development for low-emittance storage rings, and this paper attempts to focus on those topics that we think may be useful for recently started upgrade projects and for those that may come in the future.

There are several factors that can limit the performance of a storage ring, potentially preventing it from achieving its designed for beam parameters. One important target parameter that can become a limiting factor for lowemittance storage rings as well as for colliders is the beam intensity. For example, the single-bunch and the total average current can be limited by collective instabilities and/or beam-induced heating. Localized heating can be most easily avoided at high-average current if the bunch length $\sigma_{s}$ is much larger than the radius of the vacuum chamber $b, \sigma_{s} \gg b$. Many present facilities such as APS [1], NSLS-II [2], SOLEIL [3], DIAMOND [4], MAX-IV [5], etc., do not satisfy this condition, since their vacuum chamber size $b \geq 11 \mathrm{~mm}$ while the bunch length $\sigma_{s} \lesssim b$.

Many recent upgrade projects, including the ALS-U [6], APS-U [7], SIRIUS [8], PETRA-IV [9], SLS-2 [10],

Published by the American Physical Society under the terms of the Creative Commons Attribution 4.0 International license. Further distribution of this work must maintain attribution to the author(s) and the published article's title, journal citation, and DOI.
ESRF-EBS [11], and DIAMOND-II [12] do not satisfy this condition on the bunch length either. This is true even though the vacuum aperture typically decreases to enable the stronger magnetic fields required for the multiple bend achromat concept [13], because the natural bunch length in these rings also decreases. We illustrate this in Table I, where we compare the main parameters of various upgrade projects from the beam-intensity and heat-load point of view. We see that while some chamber radii may be as small as $6 \mathrm{~mm}$, and some projects have high- $(\sim 10 \mathrm{~mm})$ and low-chamber profiles $(\sim 6 \mathrm{~mm})$, the natural bunch length in all cases is typically two to four times smaller than the chamber radius. Unfortunately, such short and intense bunches typically have large Touschek and intrabeam scattering rates that result in a short lifetime and larger emittance, and furthermore can excite large wakefields around the ring which lead to higher instability growth rates and beam-induced heating. To mitigate these negative effects, all upgrade projects are looking for ways to extend the bunch length beyond its natural value $\sigma_{s 0}$.

The usual way to increase the bunch length employs a higher harmonic cavity to flatten the accelerating $\mathrm{rf}$ potential and stretch the bunch [14-28]. The total system is not trivial, and some additional effort is required to tune the rf systems in such a way that they do not disturb operation of the longitudinal and transverse bunch-bybunch feedback systems. Furthermore, predicting the longitudinal dynamics then requires full particle tracking analysis with all systems involved and including the total wakefield of the ring. Each project may choose their preferred technology for the higher harmonic cavity, and typically bunch lengthening factors of 2-5 are expected. 
TABLE I. Parameters of multibend achromat storage rings.

\begin{tabular}{lcccccc}
\hline \hline & Radius & Natural bunch length & Number of bunches & Revolution period & Average current & Coefficient \\
\cline { 2 - 7 } & $b(\mathrm{~mm})$ & $\sigma_{s 0}(\mathrm{~mm})$ & $M$ & $T_{0}(\mu \mathrm{s})$ & $I_{\text {ave }}(\mathrm{mA})$ & $T_{0} I_{\text {ave }}^{2} 10^{12} / \mathrm{M}$ \\
\hline APS-U & 11 & 4.2 & $48 / 324$ & 3.68 & 200 & $3068 / 455$ \\
ESRF-EBS & $10 / 6.5$ & 2.9 & $192 / 992$ & 2.8 & 200 & $583 / 112$ \\
ALS-U & $10 / 6.5$ & 3.5 & 284 & 0.66 & 500 & 581 \\
SLS-2 & 10 & 2.7 & 400 & 0.97 & 300 & 380 \\
DIAMOND-II & 10 & 3.2 & 900 & 1.87 & 500 & 187 \\
SIRIUS & 12 & 2.4 & 863 & 7.7 & $100 / 80$ \\
PETRA-IV & 10 & 3.3 & $960 / 80$ & 801 & $80 / 615$ \\
\hline \hline
\end{tabular}

Stretching the bunch as much as possible will significantly reduce image-current heat loads on vacuum components, which is particularly important for in-vacuum undulators and cryogenic chambers for superconducting insertion devices (IDs), since the full gap in these chambers can be as small as 4-6 $\mathrm{mm}$. In addition, bunch lengthening will result in smaller wakefield-induced heating in sensitive components such as beam position monitors (BPMs), bellows, gate valves, and stripline kickers, and may reduce the engineering requirements for flexible rf linings and gap tolerances.

In addition to the considerations of the bunch length, smaller chamber sizes make the handling of synchrotron radiation a more challenging issue. In particular, protecting sensitive components requires a variety of absorbers (masks) that may be quite close to the electron beam. Hence, their contribution to the total impedance of the ring needs to be well analyzed and optimized. In a similar manner, as the chambers become smaller the impedance cost of any small cavities between the flange joints (Fig. 1) and small bumps caused by the welding process (Fig. 2) can be significantly enhanced. The former of these can be particularly important even if the impedance per joint is

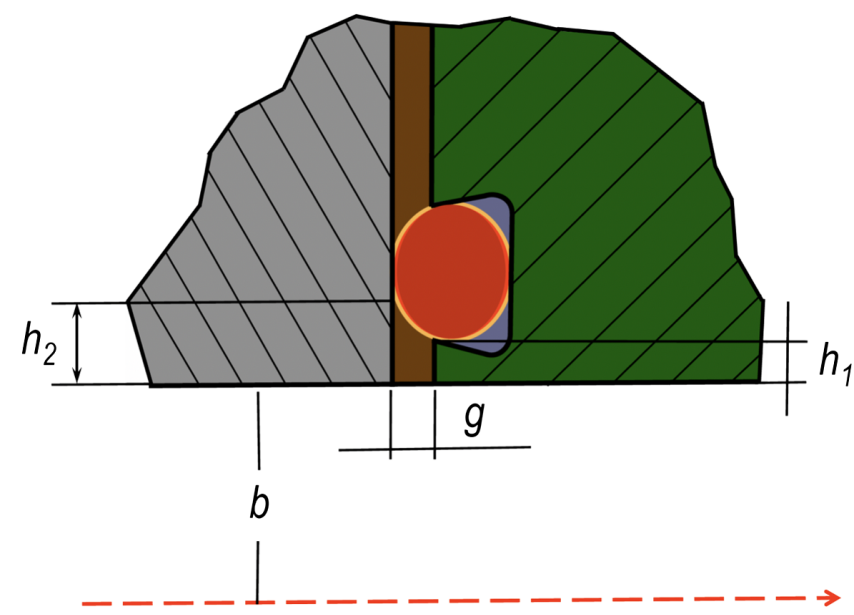

FIG. 1. Cross-section view of a flange joint with an rf contact spring between them in the trapezoidal groove. See Fig. 19 for more details. small, since impedance effects tend to sum such that hundreds or thousands of such contributions may result in significant effects.

This paper is organized as follows: we begin by introducing the basic definitions of wakefield and impedance, after which we review computer cluster considerations and requirements for numerical simulations of impedance and collective effects, and then discuss the choice of the electrodynamics software. Next, we proceed to describe how impedance simulations are done and provide a few examples illustrating how these simulations may be cross-checked with the analytical results, how geometrical variations in components can lead to important impedance consequences, and to what extent each component can be analyzed individually. These examples are meant to highlight critical components and show how their impedance can be considered. Finally, we describe how to use the computed impedance to make predictions regarding storage ring performance, including both the beam-induced heating analysis and its application to tracking studies of collective effects and instability thresholds.

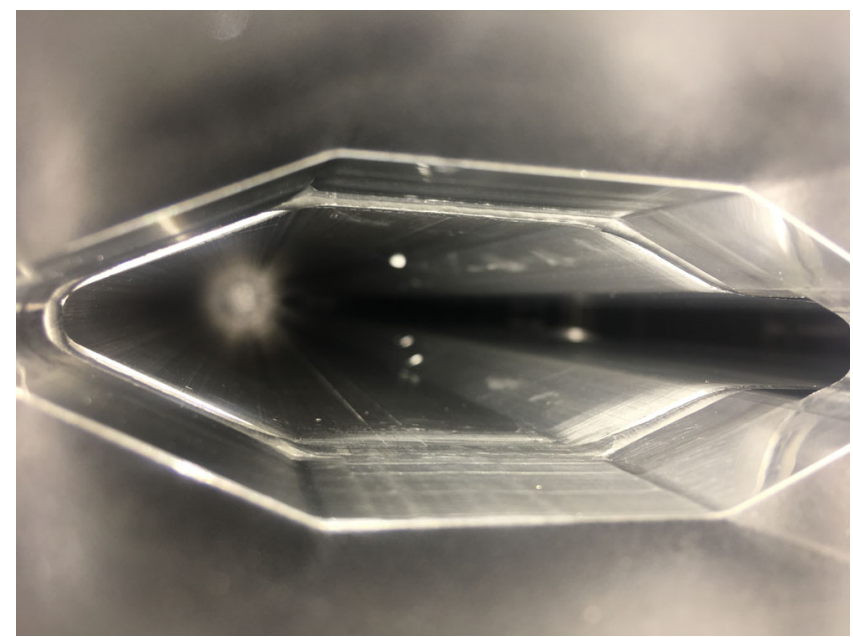

FIG. 2. Internal view of the attached flange to an $\mathrm{Al}$ vacuum chamber by welding. The internal stitch weld was cut out on the top and the bottom of the vacuum chamber, to minimize its contribution to the total impedance of the ring. 


\section{DEFINITIONS}

Wakefields quantify the impulse given by a source particle to another test particle. They are useful to describe collective interactions in an accelerator, since these forces are typically sufficiently weak and/or localized such that the relative positions between particles do not significantly change during their interaction. Under this assumption the change in test particle energy is given by integrating the longitudinal electric field $E_{\|}$over space, and we can define the longitudinal wakefield $\mathcal{W}_{\|}(x, y, s)$ via

$$
\begin{aligned}
\Delta \gamma & =\frac{e}{m c^{2}} \int_{\infty}^{\infty} d \tau E_{\|}(x, y, s ; \tau) \\
& =\frac{e^{2}}{m c^{2}} \mathcal{W}_{\|}(x, y, s),
\end{aligned}
$$

where $s$ is $c$ times the relative arrival time between the source and test particle, $\tau$ is the distance along the trajectory, and $c, e$, and $m$ are the speed of light, the electron charge, and its mass, respectively. In the ultrarelativistic limit causality insures that the wakefield vanishes in front of the source, so that $\mathcal{W}_{\|}(x, y, s<0)=0$ for ultrarelativistic point particles.

Wakefields can describe direct space-charge forces and radiation forces resulting from accelerated charges, but here we focus on wakefields that arise from the electromagnetic interaction of a particle with the chamber wall. These wakefields are typically divided into their resistive and geometric components: the former arise because the chamber walls are not perfect conductors, while the latter are generated whenever the boundary conditions at the walls change due to changes in chamber geometry. We will assume that the chamber dimensions are much larger than the beam transverse extent, over which distances $\mathcal{W}_{\|}(x, y, s) \approx \mathcal{W}_{\|}(0,0, s)=W_{\|}(s)$; Following usual practice, in the following we will refer to $W_{\|}(s)$ as the longitudinal wakefield.

In analogy with Eq. (1), the transverse wakefield is defined in terms of the transverse impulse via

$$
\begin{aligned}
\Delta \boldsymbol{x}^{\prime} & =\frac{e}{\gamma m c^{2}} \int_{\infty}^{\infty} d \tau(\boldsymbol{E}(x, y, s ; \tau)+\boldsymbol{v} \times \boldsymbol{B}(x, y, s ; \tau)) \\
& =-\frac{e^{2}}{\gamma m c^{2}} \mathcal{W}_{\perp}(x, y, s) .
\end{aligned}
$$

When the particles are near the reference orbit, we can again Taylor expand the wakefield. In this case, the expansion depends upon the transverse offset of both the source particle $\boldsymbol{x}_{s}$ and the test particle $\boldsymbol{x}_{t}$, and for the horizontal plane, we write

$$
\mathcal{W}_{x}(x, y, s) \approx W_{x}^{M}(s)+x_{s} W_{x}^{D}(s)+x_{t} W_{x}^{Q}(s),
$$

where $W_{x}^{M}$ is the monopole component, $W_{x}^{D}$ is the dipole wakefield, and $W_{x}^{Q}$ is the quadrupolar wakefield—similar expressions apply along $y$. If the chamber is left-right and top-bottom symmetric $W_{\perp}^{M}=0$, while $W_{\perp}^{Q}$ vanishes in axially symmetric structures. Furthermore, since $\boldsymbol{W}_{\perp}^{D}$ is the dominant driver of transverse instabilities, the dipole wakefield is often simply referred to as the transverse wakefield.

The total energy loss (or total kick) is obtained by summing the contributions from all source particles. For example, if the beam has $N_{p}$ particles that are Gaussian distributed with rms length $\sigma_{s}$, then the energy loss of a particle located at position $s$ is

$$
\Delta \gamma=\frac{e^{2} N_{e}}{m c^{2}} \int d s^{\prime} W_{\|}\left(s-s^{\prime}\right) \frac{e^{-s^{\prime 2} / 2 \sigma_{s}^{2}}}{\sqrt{2 \pi} \sigma_{s}} .
$$

Similar expressions apply for the transverse kick. Hence, the point-particle wakefields $W_{\|}$and $\boldsymbol{W}_{\perp}$ serve as Green functions for the longitudinal and transverse impulses.

Finally, since the force is in the form of the convolution (4), it is often useful to consider the Fourier transform of the wakefields, namely, the impedance. Indeed, in Fourier space Eq. (4) can be interpreted as the familiar relation $V(\omega)=Z(\omega) I(\Omega)$, with the impedances

$$
\begin{aligned}
Z_{\|}(\omega) & =\frac{1}{c} \int d s e^{i \omega s / c} W_{\|}(s), \\
Z_{x, y}(\omega) & =\frac{i}{c} \int d s e^{i \omega s / c} W_{x, y}(s) .
\end{aligned}
$$

\section{ELECTROMAGNETIC SIMULATIONS}

The most comprehensive approach to estimate instability thresholds for accelerator projects is to carry out computer simulation studies that track particles through the ring in the presence of all the relevant wakefields (or, equivalently, the impedances). The wakefields are in turn computed from each vacuum component that is seen by the beam. These vacuum components may have complex three-dimensional (3D) geometries, and part of the design process should include minimizing the impedance as much as possible while satisfying other design constraints, e.g., space and cost. Hence, some effort should be spent calculating the impedance and verifying that it satisfies requirements. There are several two-dimensional (2D) and 3D electromagnetic codes that can compute wakefields and/or impedances by simulating the interaction of a charged particle beam with the vacuum chamber in the time-domain and/or frequency-domain. These codes include GdfidL [29], CST Particle Studio [30], HFSS [31], ECHOz1/z2 [32,33], ECHO 3D [34], URMEL [35], Vorpal [36], ACE3P [37], ABCI [38], and Poisson/Superfish [39].

Each code has its advantages and disadvantages, and everyone must decide for themself which simulation package to use. Some factors that one may consider for code 
selection were presented in Refs. [40,41], where the authors made a detailed comparison between several time-domain codes using an example tapered transition structure that is common in accelerators. Part of the selection process should consider its ability to use parallel computing resources, particularly if one plans to calculate the impedance of complex geometries in 3D.

Regardless of the code chosen, an important part of wakefield simulation is determining the accuracy and numerical resolution required for a particular component. Codes typically calculate the wakefield from a Gaussian bunch of rms length $\bar{\sigma}_{s}$, so that the numerical mesh needs to be small enough to resolve both $\bar{\sigma}_{s}$ and the geometrical features of the chamber under consideration [42]. Furthermore, when the wakefield is to be used as a pseudo-Green function in tracking simulations that determine how collective effects impacts the dynamics, one typically needs to properly identify $\bar{\sigma}_{s}$. Simulations designed to calculate rf heating and/or evaluate low-frequency modes may calculate the wakefield using a source current whose length equals that of the bunch in the storage ring, $\bar{\sigma}_{s}=\sigma_{s 0}$. On the other hand, one would like the wakefield to suitably approximate that of a point charge when predicting collective instabilities using tracking simulations. In this case, determining the appropriate $\bar{\sigma}_{s}$ is less straightforward. The general rule of thumb for tracking is that the wakefield should be calculated with a source current whose length is ten times smaller than that of the equilibrium beam, $\bar{\sigma}_{s} \approx \sigma_{s 0} / 10$. In our experience, this guideline gives a reasonable estimate of the required $\bar{\sigma}_{s}$, although we have concluded that the actual requirements are set by the impedance under consideration. Specifically, we observe in Sec. VII that $\bar{\sigma}_{s}$ must be small enough to fully resolve the frequency of the first large, resonancelike feature of the impedance. Unfortunately, this requirement is not fully prescriptive in that it does not precisely define the resonance. Nevertheless, we have found it helps explain why values of $\bar{\sigma}_{s}$ between $\sigma_{s 0} / 10$ and $\sigma_{s 0} / 15$ give convergent predictions in particle tracking.

As an example of how mesh size (and, hence, simulation time) can affect accuracy, in Fig. 3, we show results of a study of the NSLS-II synchrotron radiation flange absorber. The collimator-type geometry is shown at the top, where we see that the flange absorber transitions from the nominal octagonal chamber dimensions with a $76 \mathrm{~mm}$ full horizontal width and a $25 \mathrm{~mm}$ full vertical height to one with a $64 \mathrm{~mm}$ width and $21 \mathrm{~mm}$ height. The next two plots compare the numerically simulated wakefields and the imaginary part of the longitudinal impedance using a $\Delta=$ $50 \mu \mathrm{m}$ and $\Delta=20 \mu \mathrm{m}$ stepsize. We see that obtaining accurate results for a bunch length $\bar{\sigma}_{s}=0.3 \mathrm{~mm}$ requires a GdfidL mesh spacing $\Delta \lesssim \bar{\sigma}_{s} / 15$, and this resolution is crucial if we want to accurately model the impedance for frequencies beyond $75 \mathrm{GHz}$. The ЕсHO 3D code calculates the wakefield using the indirect integration method, which typically requires a less stringent mesh
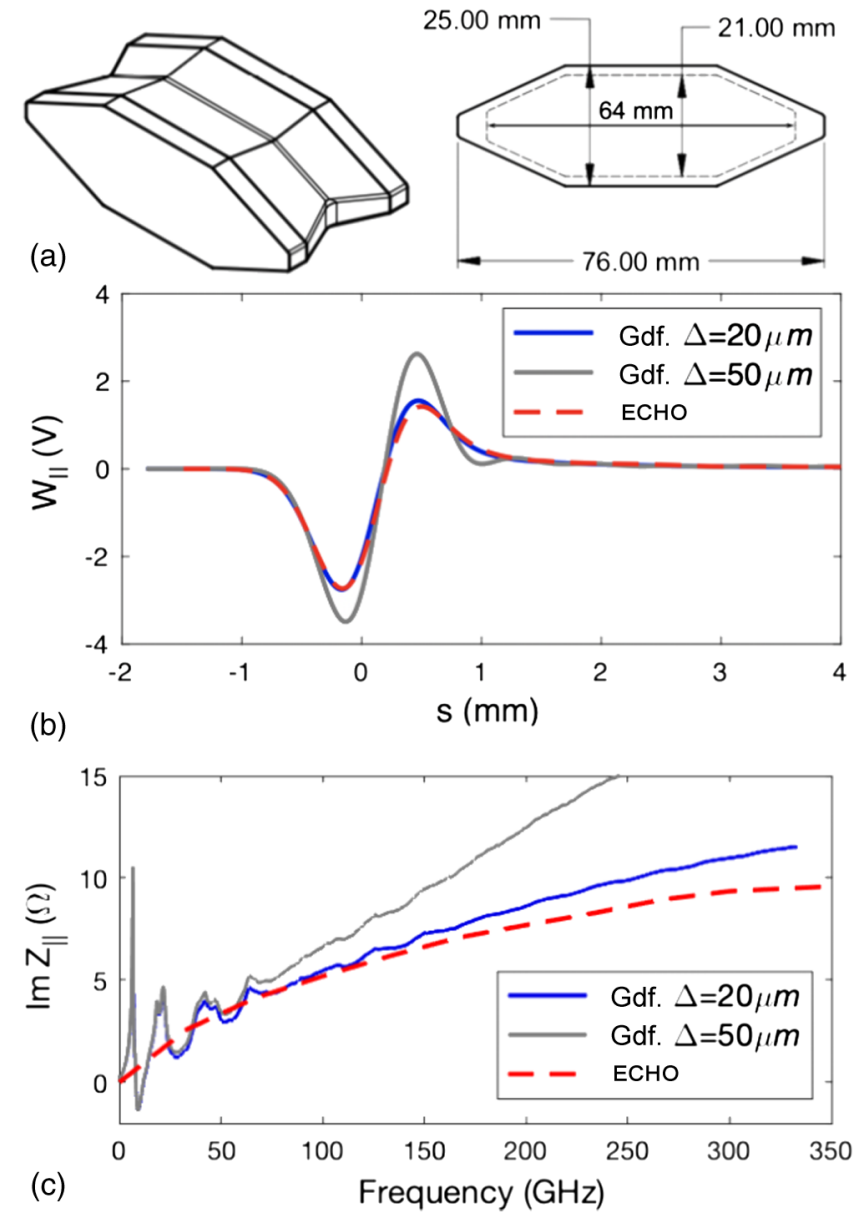

FIG. 3. (a) Flange absorber and its cross-section view, showing how the chamber aperture reduces from $76 \times 25 \mathrm{~mm}$ to $64 \times$ $21 \mathrm{~mm}$ over a $12 \mathrm{~mm}$-long tapered transition. (b) Comparison of the flange longitudinal wakefield computed by the GdfidL code using a $\Delta=50 \mu \mathrm{m}$ (gray) and $\Delta=20 \mu \mathrm{m}$ stepsize (blue) with that of the ECHO 3D code (red dashed). (c) Calculated imaginary part of the longitudinal impedance for the same cases.

spacing of $\Delta \lesssim \bar{\sigma}_{s} / 5$ for reliable results. The apparent discrepancy between the code predictions at low frequencies is an artifact of the different wakefield lengths used for the two simulations: the GdfidL wakefield is $s=5 \mathrm{~m}$ long so that its frequency resolution is $\Delta f=c / s_{\max } \approx 60 \mathrm{MHz}$, while the ECHO 3D longitudinal wakefield was only calculated up to $s=7.5 \mathrm{~mm}$. Hence, ECHO samples the impedance every $40 \mathrm{GHz}$, and the resonances at low frequency are not visible.

One method that can potentially reduce the required computational resources is to use a moving window algorithm $[29,43,44]$. In this scheme, the computational volume moves with the source current and only the wakefield that is "in" the window is computed. The moving window algorithm can be advantageous when simulating short-range wakefields over distances in $s$ that are less than that of the vacuum component under consideration, since in this case the required memory can be reduced by the ratio of the wake to structure length. As an example, using 
GdfidL to simulate the $150 \mathrm{~mm}$-long NSLS-II rf shielded bellows with a $\Delta=30 \mu \mathrm{m}$ stepsize and over a wakefield length of $s=3 \mathrm{~mm}$ requires $218 \mathrm{~GB}$ RAM for the "standard" finite-difference time domain algorithm, while the moving window needs only 14 GB of RAM. This memory difference becomes smaller as the wakefield length increases, and whether the moving window provides a memory or computational time advantage depends upon the code under consideration. We have found that GdfidL's moving window algorithm (windowwake) is typically not advantageous for the $\gtrsim 100 \mathrm{~mm}$ wakefield lengths required for most storage ring applications. On the other hand, some codes (including ECHO) are written to naturally employ a moving window.

Finally, a potential shortcoming of finite-difference algorithms is numerical dispersion [42], in which the discretized mesh modifies the electromagnetic dispersion relation in unphysical ways that depend upon the mesh size. Developing algorithms that are (nearly) dispersion-free over some angular and/or spectral range is not an easy task. It should be noted here that some of the most popular 3D electromagnetic codes, including GdfidL and CST Particle studio, are commercial. Since the GdfidL code has the ability to perform parallel computing and has demonstrated reliable results for geometries with different complexities, both the APS and NSLS-II have used this code as the main tool for the wakefield simulations since the early 2000s.

Numerically calculating wakefields and impedances in complex structures can require significant computational resources. Hence, the needs of wakefield calculations should be added to those of lattice optimization, beam dynamics simulations, etc., when obtaining the required computational resources. If this involves building a dedicated cluster, the first step is to consider the maximum planned capacity so that space with enough cooling capacity and power supplies can be reserved. Two basic models to acquiring the desired hardware can then be considered: the first option is to attempt to purchase the entire cluster at once as was done for significant parts of the APS-U project at ANL; The second option is to buy a rack with a couple of nodes and a fast switch, to which additional nodes are added as funds become available over the next several years, as was done for the NSLS-II accelerator physics cluster.

Ultimately, the number of multicore processors and the total RAM memory drives the cluster cost. For this reason, it is often beneficial to install nodes that have different computing capabilities tailored to differing needs. As an example, simulations of lattice dynamics do not require significant memory or communication between processors, so that increasing the number of multicore processors strongly improves the performance. On the other hand, electromagnetic simulations to obtain wakefields and impedances require more communication between nodes and are typically RAM hungry, particularly when one considers high-resolution wakefields for complex 3D geometries. In this case, the minimum amount of required RAM memory can be estimated as minimum 4 GB per core (task), although more can be quite beneficial.

\section{IMPEDANCE MODELING}

We believe that the impedance analysis should begin in the early stages of any accelerator upgrade project, so that it can help inform the design of vacuum components. Even when the detailed vacuum system is not known, a preliminary impedance lattice and/or budget can be estimated using simplified models of key vacuum chamber components. Such approximate models can identify the major sources of impedance early in the design phase, so that particular attention can be made to the impedance cost as the design matures.

We have found it useful to summarize the relative resistive and geometric contributions of the developing impedance model within a single file that lists all impedance elements including its material, length, location in the ring, internal dimensions, the computed loss factor, low frequency $\Im\left(Z_{\|}\right) / n$, and the betatron function-weighted kick factor. In the early stages of ring design, these quantities can give rough assessments of rf heating and instability thresholds. For example, the NSLS-II's initial estimate for the microwave instability [2] applied Oide and Yokoya's formula [45]

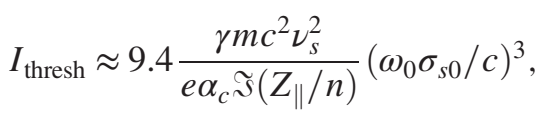

where $\nu_{s}$ is the synchrotron tune and $\alpha_{c}$ is the momentum compaction. Equation (7) has the same form as Boussard's extention [46] to the Keil-Schnell formula but with the factor 9.4 replacing Boussard's $\sqrt{2 \pi} \approx 2.5$. Using Eq. (7) with a $\Im\left(Z_{\|} / n\right)=0.4 \Omega$ that was based upon published results at the APS and ESRF, the first estimate for the microwave instability threshold was $0.6 \mathrm{~mA}$. This turned out to be about half of the final measured value of $1.2 \mathrm{~mA}$.

Once a more detailed design of the vacuum system develops, however, the loss factor, $\Im\left(Z_{\|} / n\right)$, and kick factor are insufficient for detailed predictions of collective effects. Nevertheless, these quantities do provide a quick and easy way to gauge the relative impedance cost of various components and can be particularly useful as a metric for how the impedance contribution of any particular component may change as the design matures.

We show an example from the summary that has been developed at NSLS-II in Fig. 4. The main part describes all the components in the identical long straight sections of cells $6,14,20$, and 26. Various abbreviations have been used in the table for the names of vacuum components and the resistive wall surfaces, like bellows (BLW) with stainless steel (SS), silver coated (Ag) and GlidCop 


\begin{tabular}{|c|c|c|c|c|c|c|c|c|c|c|c|}
\hline C249 & $\stackrel{\Delta}{\vee} \vee \vee f x$ & FABS & & & & & & & & & \\
\hline 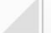 & C & D & $E$ & $\mathrm{~F}$ & G & $\mathrm{H}$ & 1 & $J$ & $\mathrm{~K}$ & L & \\
\hline 1 & & & & & & & & & & & \\
\hline 2 & & \multicolumn{5}{|c|}{ Cells: $6,14,20$ and 26} & & & & & \\
\hline 3 & & & & & & & & & & & \\
\hline 4 & From left to right & $\mathrm{S}(\mathrm{mm})$ & $\operatorname{betax}(m)$ & $\operatorname{betay}(\mathrm{m})$ & $\mathrm{L}(\mathrm{mm})$ & $\mathrm{L}(\mathrm{mm})^{\star} 4$ & RW & Conductivity & Radius (ry), m & $\mathrm{s} 0$ & $k$ \\
\hline 5 & Fast Corrector & 48533.65 & 21.35395438 & 8.764469694 & 168.5 & 674 & Inc & 775194 & 0.0125 & 0.00010226 & \\
\hline 6 & BLW5(SS) & 48702.15 & 21.28513577 & 8.346447559 & 5.540 & 22.16 & ss & 1350000 & 0.0125 & 8.49962E-05 & \\
\hline 7 & BLW5(GCu) & 48707.69 & 21.28292024 & 8.332989857 & 52.310 & 209.24 & $\mathrm{Cu}$ & 54050000 & 0.0127 & 2.51097E-05 & \\
\hline 8 & BLW5(Ag) & 48760 & 21.26214855 & 8.206817181 & 69.170 & 276.68 & $\mathrm{Ag}$ & 63000000 & 0.0125 & $2.36082 E-05$ & \\
\hline 9 & CHM1 & 48829.17 & 21.23509252 & 8.042471831 & 3956.3 & 15825.2 & Al & 31600000 & 0.0125 & $2.97131 \mathrm{E}-05$ & \\
\hline 10 & BLW6(SS) & 52785.47 & 20.46575214 & 3.369298068 & 5.540 & 22.16 & sS & 1350000 & 0.0125 & 8.49962E-05 & \\
\hline 11 & BLW6(GCu) & 52791.01 & 20.46574729 & 3.369268602 & 77.710 & 310.84 & $\mathrm{Cu}$ & 54050000 & 0.0127 & 2.51097E-05 & \\
\hline 12 & BLW6(Ag) & 52868.72 & 20.46599535 & 3.370775402 & 69.170 & 276.68 & $\mathrm{Ag}$ & 63000000 & 0.0125 & 2.36082E-05 & \\
\hline 13 & CHM2 & 52937.89 & 20.46671258 & 3.375132019 & 3956.2 & 15824.8 & Al & 31600000 & 0.0125 & $2.97131 \mathrm{E}-05$ & \\
\hline 14 & BLW5(SS) & 56894.09 & 21.28587228 & 8.350921296 & 5.540 & 22.16 & SS & 1350000 & 0.0125 & 8.49962E-05 & \\
\hline 15 & BLW5(GCu) & 56899.63 & 21.28809181 & 8.364403268 & 52.310 & 209.24 & $\mathrm{Cu}$ & 54050000 & 0.0127 & 2.51097E-05 & \\
\hline 16 & BLW5(Ag) & 56951.94 & 21.30919695 & 8.492601424 & 69.170 & 276.68 & $\mathrm{Ag}$ & 63000000 & 0.0125 & $2.36082 E-05$ & \\
\hline 17 & Fast Corrector & 57021.11 & 21.33751506 & 8.664612992 & 168.5 & 674 & Inc & 775194 & 0.0125 & 0.00010226 & \\
\hline 18 & & & & & 8655.96 & 34623.84 & & & & & \\
\hline 19 & & & & & & & & & & & \\
\hline 20 & & & & & & & & & & & \\
\hline
\end{tabular}

FIG. 4. A spreadsheet summary of the impedance contributions with relevant lattice parameters. Component differences amongst the various sectors are tracked and updated as changes are made. BLW is the bellows and CHM is the standard vacuum chamber.

$(\mathrm{GCu})$ surfaces, and standard vacuum chamber (CHM) with aluminum $(\mathrm{Al})$.

The next two sections further discuss the modeling of such vacuum components, paying particular attention to first the resistive wall contribution that comes about from losses in the chamber walls, and then to the geometric contribution that arises from changes in the chamber cross section. While some electrodynamics codes can include both the geometric and resistive effects in their simulations, we have found that it is often simpler to compute these two sources separately. This approach of separating the two impedance contributions has been employed by, e.g., the NSLS-II, APS-U [47], ALS-U, and EIC [48] projects.

\section{A. Resistive wall}

Vacuum chamber components with finite conductivity interact with the beam to produce both longitudinal and transverse forces. In simple chamber geometries, these forces can be computed analytically, from which longitudinal and transverse wakefields and/or impedances may be derived. Our typical approach to computing the resistive wall wakefield is to model each individual component as either a circular chamber or as two parallel plates, and then apply the analytic wakefields/impedances derived by Piwinski [49], Bane and Sands [50], and Yokoya [51]. The circular model can be used when the chamber is approximately azimuthally symmetric, while the parallel plates model applies when the aspect ratio between the horizontal and vertical dimensions becomes large. Calculations for rectangular and elliptical chambers show that the longitudinal resistive force doesn't vary much between these two extremes, while the transverse force is largest for round chambers, and smoothly transitions to the parallel plate limit as the ratio between the two dimensions becomes larger than 2 to 3 .
The longitudinal resistive wall force is the same in both round and flat chambers. The longitudinal wakefield driven by a $N_{p}$ particles that are Gaussian distributed with rms width $\bar{\sigma}_{s}$ is

$$
\begin{aligned}
W_{\|}^{\mathrm{rw}}(s)= & \frac{r_{e} m c^{2} N_{p}}{2 b \sqrt{2 \mu_{r} Z_{0} \sigma_{\mathrm{con}}}}\left|\frac{s}{\bar{\sigma}_{s}}\right|^{3 / 2} e^{-s^{2} / 4 \bar{\sigma}_{s}^{2}} \\
& \times\left\{I_{1 / 4}\left(s^{2} / 4 \bar{\sigma}_{s}^{2}\right)-I_{-3 / 4}\left(s^{2} / 4 \bar{\sigma}_{s}^{2}\right)\right. \\
& \left.-\operatorname{sgn}(s)\left[I_{-1 / 4}\left(s^{2} / 4 \bar{\sigma}_{s}^{2}\right)-I_{3 / 4}\left(s^{2} / 4 \bar{\sigma}_{s}^{2}\right)\right]\right\},
\end{aligned}
$$

where $r_{e}$ is the classical electron radius, $Z_{0} \approx 120 \pi \Omega$ is the impedance of free space, $b$ is the chamber radius or half height, $\sigma_{\mathrm{con}}$ is the chamber's electrical conductivity, and $\mu_{r}$ is its relative permeability.

The vertical resistive wall wakefield was derived analytically for round and flat vacuum chambers by A. Piwinski [49,52]. Bane and Sands [50] demonstrated that Piwinski's result is valid for a long Gaussian bunch whose rms width satisfies $\sigma_{s} \gg s_{0}=\left(2 b^{2} / Z_{0} \sigma_{\text {con }}\right)^{1 / 3}$, a condition that is typically well satisfied in storage rings. Under these conditions, the radial force generated by a beam that is displaced from the axis of a round chamber by an amount $r_{o}$ is given by [52]

$$
\begin{aligned}
W_{\perp}^{\mathrm{rnd}}(s)= & \frac{2 r_{e} m c^{2} N_{p}}{b^{3} \sqrt{2 \mu_{r} Z_{0} \sigma_{\mathrm{con}}}} \frac{r_{o} e^{-s^{2} / 4 \bar{\sigma}_{s}^{2}}}{\left(1-r_{o}^{2} / b^{2}\right)^{2}}\left|\frac{s}{\sigma_{s}}\right|^{1 / 2} \\
& \times\left[I_{-1 / 4}\left(s^{2} / 4 \bar{\sigma}_{s}^{2}\right)-\operatorname{sgn}(s) I_{1 / 4}\left(s^{2} / 4 \bar{\sigma}_{s}^{2}\right)\right] .
\end{aligned}
$$

The force diverges as the beam approaches the wall and $r_{o} \rightarrow b$, while for small displacements the force scales linearly with $r_{o}$ and we can approximate it using the dipolar approximation $W_{\perp} \approx r_{o}\left(\partial W_{\perp} / \partial r_{o}\right)_{r_{o}=0}=r_{o} W_{\perp \text {,dip. }}$. 
The transverse force for a beam that is displaced in a chamber composed of two flat plates was derived in Ref. [49]. The expression for an arbitrary offset is similar in form to that of Eq. (9). Here, we just give the dipolar component of the force along the beam axis that is relevant for small offsets in a chamber of half height $h$ :

$$
\begin{aligned}
W_{\perp, \text { dip }}^{\text {flat }}= & \frac{\pi^{2} r_{e} E_{0} N_{p}}{6 h^{3} \sqrt{2 \mu_{r} Z_{0} \sigma_{\text {con }}}}\left|\frac{s}{\bar{\sigma}_{s}}\right|^{1 / 2} e^{-s^{2} / 4 \bar{\sigma}_{s}^{2}} \\
& \times\left[I_{-1 / 4}\left(s^{2} / 4 \bar{\sigma}_{s}^{2}\right)-\operatorname{sgn}(s) I_{1 / 4}\left(s^{2} / 4 \bar{\sigma}_{s}^{2}\right)\right] .
\end{aligned}
$$

Comparing this to the dipole expression for the round chamber, we see that the dipole wakefield for round and flat chambers with identical minimum gaps, $b=h$, are related by

$$
W_{\perp, \text { dip }}^{\text {flat }}(s)=\frac{\pi^{2}}{12} W_{\perp, \text { dip }}^{\mathrm{rnd}}(s) .
$$

The previous considerations apply to conductive metals but modern storage rings often applying thin coatings to certain chambers to improve their performance. For example, ceramic chambers used for fast kicker chambers typically have thin layers of metal applied to improve their impedance, while a thin coating of nonevaporable getter (NEG) or similar material has been increasingly employed in small aperture chambers to improve their vacuum performance. We briefly summarize impedance considerations for these chambers below.

Highly conductive chambers are inappropriate in areas where magnetic fields vary rapidly with time, because the transient eddy currents supported by good conductors will shield part of the magnetic field, resulting in delayed and distorted magnetic waveforms. Nevertheless, such chambers are often coated with a thin conducting layer to prevent damage and vacuum failure from image currents in the beam. For example, there are five ceramic kicker chambers in the NSLS-II storage ring, all of which are coated with Titanium. However, coating ceramics in complex geometries is a difficult task and verifying the coating thickness and uniformity is technically challenging. Several light sources, including DIAMOND [53], Pohang Light Source [54], and NSLS-II [55] reported overheating of their ceramics chambers, which was believed to have been caused from some combination of poor coating adhesion and lack of coating uniformity with appropriate thickness. Predicting the impedance of even ideal examples of such layered chambers has had a complicated past. For example, the multilayer approach first derived by Piwinski [56] provides an analytic solution to the problem, but this solution does not apply in the limit of very thin coatings, since his power loss formula, Eq. (18) in Ref. [56], incorrectly vanishes in the limit that the coating thickness goes to zero. Hence, to determine the performance of the thin coatings that are typically required for accelerator applications, one must turn to numerical field matching techniques as is done in, for example, the Impedancewake2D code [57] developed at CERN.

Finally, there is also a long history that has applied field matching techniques to compute the impedance in a variety of other multilayered chambers with linear permeabilities (see, e.g., [58]). Relatively simple analytic expressions for two-layered chambers that apply to storage ring parameters are available in, e.g., Ref. [59], while more complicated cases can again be numerically evaluated using the Impedancewake2D code [57]. The essential physics can be estimated by comparing the coating thickness to the skin depth $\sqrt{2 c /|\omega| \sigma_{\operatorname{con}} Z_{0}}$ at the frequency of interest: if the coating is much thicker than the skin depth, the behavior is dominated by the coating material, while in the opposite limit the fields predominantly see the inner layer. While the physics is fairly well understood, characterizing the electrical parameters of complex, multimaterial coatings can be quite challenging. For example, while recent measurements of NEG coatings have significantly improved our understanding of NEG conductivity [60], there are also indications that the impedance may depend upon the coating uniformity and deposition technique.

\section{B. Geometric impedance}

Strong interaction with other physicists, engineers, and technicians in the vacuum, diagnostic, and rf groups is key to successfully integrating impedance considerations in vacuum component design. It may take several iterations with these groups before all requirements are met and the design is finalized. As part of this process, the coupling impedance is calculated for each individual vacuum component assuming that there is no cross talk between the neighbor components. In this case, the total wakefield/ impedance for the ring is a sum of the individual contributions. Here, we describe several examples of wakefield and impedance analysis for individual components. We begin with two preliminary examples where analytic results are used to verify the code predictions, then continue by describing how variations in geometry can affect the generated wakefields, and finally conclude by showing two contrasting examples when interference/cross talk can be an issue with when it is not.

\section{Verifying code predictions with theory}

There are many analytic impedance results for idealized geometries that can be used to both estimate the impedance and to cross-check numerical simulations of various components. These results are typically restricted to either high or low frequencies, and may be summarized in terms of the loss factor $k_{\|}\left(\sigma_{s}\right)$ or the kick factor $k_{\perp}\left(\sigma_{s}\right)$ [61-64]. In this section, we illustrate the utility of such analytic expressions using two examples: the first is a simple step collimator, while the second is for a stripline kicker used in the NSLS-II. 
One test structure that we have found may be useful to numerically verify an electromagnetic code is that of an axially symmetric step collimator. Furthermore, this simple structure can also give a reasonable first estimate to the wakefields resulting from particle collimators or scrapers that are used in a real machine. For a cylindrical pipe that makes a sudden transition from its nominal radius $d$ to a smaller radius $b$ and then back again, the analytical expression for the high-frequency impedance derived by Kheifets [58] is

$$
Z_{\|}^{\text {step }}(k \gg 1 / b)=\left(Z_{0} / \pi\right) \ln (d / b),
$$

assuming that $k b<\gamma$. Later, Stupakov, Bane, and Zagorodnov showed [65] that the high-frequency limit where Eq. (12) applies corresponds to the optical regime, in which the field propagation can be approximated along straight lines in analogy with ray optics.

Figure 5 shows the real and imaginary parts of the impedance calculated by GdfidL for a step collimator with $b=5 \mathrm{~mm}$ and $d=25 \mathrm{~mm}$. The longitudinal wakefield was determined using a $0.3 \mathrm{~mm}$ bunch length, and we find that the impedance is approximately equal to its theoretical value $Z_{\|}^{\text {step }}=193 \Omega$ for frequencies $f>50 \mathrm{GHz} \approx 5 c / 2 \pi b$.

Our next example of code verification uses a more realistic geometry, namely, a stripline kicker that has two electrodes spanning $90^{\circ}$ each; this geometry was analyzed in more detail in Ref. [66]. While the impedance over the full frequency range must be numerically calculated, at low
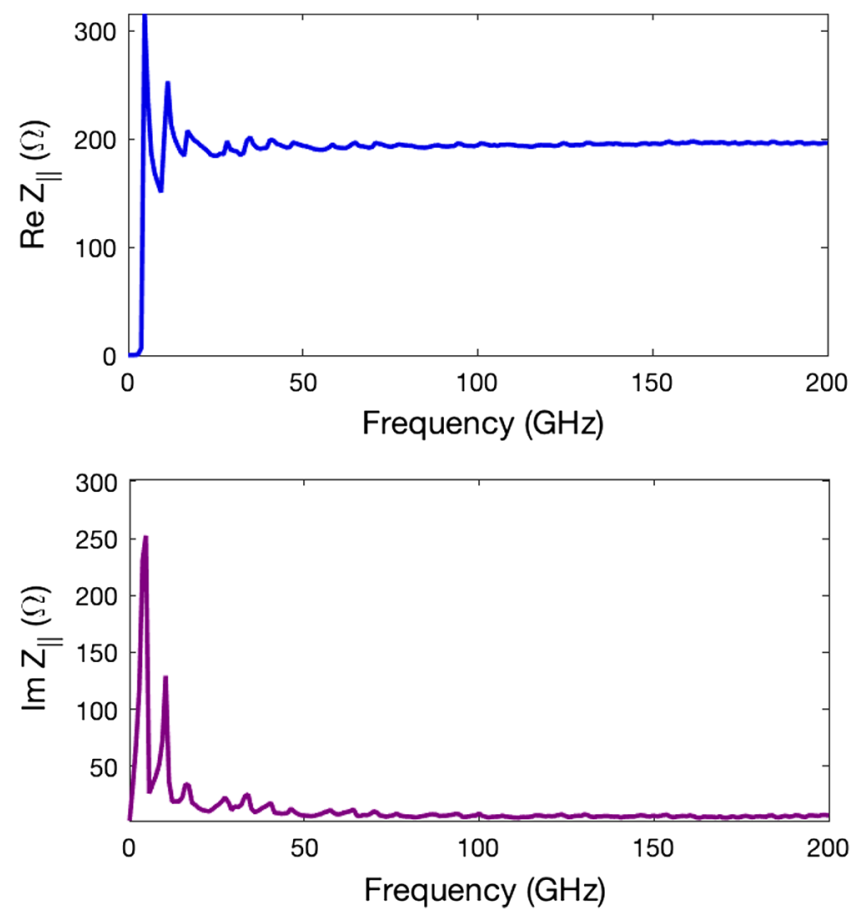

FIG. 5. Real (top) and imaginary (bottom) part of the longitudinal impedance for the step transition. frequencies we can compare numerical predictions to theory. Following Lambertson's formalism [67], the stripline longitudinal beam impedance at low frequency is

$$
Z_{\|}(k)=g_{\|}^{2} Z_{c h, \|}\left[\sin ^{2}(k L)-i \sin (k L) \cos (k L)\right],
$$

where $g_{\|}$is the longitudinal geometric factor, $Z_{c h, \|}$ is the longitudinal characteristic impedance, $k$ is the wave number, and $L$ is the electrode length. The geometric factor can be estimated using the $\mathrm{Ng}$ approximation [68]

$$
g_{\|}=\phi / \pi,
$$

while $Z_{c h, \|}$ can be approximated by

$$
Z_{c h, \|}=Z_{c x l} / \sqrt{g_{\|}}=\frac{Z_{0}}{2 \pi} \frac{\ln (d / b)}{\sqrt{g_{\|}}},
$$

where $b$ is the radius up to the electrode edge while $d$ is the vacuum chamber radius. Using the data from Ref. [69], the angle $\phi=90^{\circ}$ implies that the longitudinal geometric factor is $g_{\|}=1 / 2$, while $b=27.2 \mathrm{~mm}, d=39.6 \mathrm{~mm}$, and $L=310 \mathrm{~mm}$ gives a characteristic impedance $Z_{c h, \|}=31.87 \Omega$. It should be noted that the $g_{\|}$computed using this simple approximation is somewhat less than the $g_{\|}=0.77$ obtained numerically in Ref. [66] using the Poisson code.

We use the more accurate result to evaluate the product $g_{\|}^{2} Z_{c h, \|}=18.4 \Omega$ for Eq. (13), and compare the theoretically computed longitudinal impedance to that obtained numerically for the stripline kicker in Fig. 6. The real 3D stripline structure has been numerically computed by the GdfidL code (Fig. 6, blue traces), while the red traces are the data obtained using the analytical approach of Eqs. (13), (14), and (15). We see that the analytic formula provides a very good prediction at low frequencies, and the difference between it and the simulation results increase as $f$ increases. The theory become qualitatively incorrect when $f \gtrsim 3 \mathrm{GHz}$, in which case the frequency is larger than the cutoff frequency of the first fundamental longitudinal $E_{01}$ mode and Eq. (13) no longer applies.

\section{Effects of tolerance and variations in geometry}

Wakefield analysis typically starts with idealized version of components and then is refined to the point of simulating wakefields directly from Computer Aided Design (CAD)-rendered drawings. Nevertheless, installed components may be somewhat different from the designs due to manufacturing tolerances or installation precision, and may even differ in unexpected ways. Here, we illustrate how these issues may affect the impedance using two examples: the first uses an example from the APS to show how small geometry changes can have a big impact on the impedance if it is close to the particle beam. The second illustrates how installation 

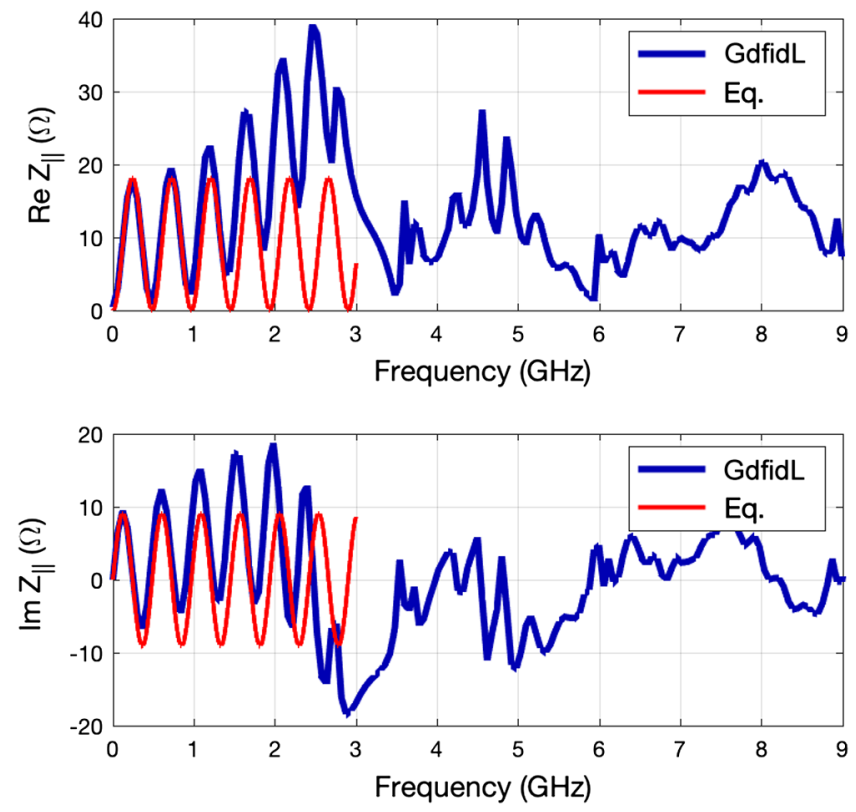

FIG. 6. Real (top) and imaginary (bottom) part of the longitudinal impedance for two $90^{\circ}$ electrode angle. The blue trace is the GdfidL numerically simulated result. The red trace is the analytical approximation of Eq. (13).

variations may affect wakefield performance. In an ideal world, one would identify and analyze all such effects and take them into account during design, but this is, in our opinion, unrealistic. Nevertheless, we think that these examples show that one should be particularly careful in the design of components that are close to the beam and expected to generate fairly large wakefields, and furthermore that some tolerance studies of critical components should be done to anticipate how installation variations may affect rf heating.

Transitions to and from narrow gap IDs can be a significant source of transverse impedance at storage ring light sources. At the APS, the vertical gap of the vacuum chamber reduces from its nominal $42 \mathrm{~mm}$ to a minimum between 5 and $8 \mathrm{~mm}$ depending upon the ID, such that approximately one-third of the vertical impedance is due to ID transitions themselves, while nearly another third is due to the resistive wall in the narrow gap ID chamber. To reduce the impedance of the transitions, Y.-C. Chae numerically optimized a two-taper scheme, with the resulting geometry shown in Fig. 7(a). This design reduces the impedance partly because of its larger slope at larger aperture, and partly by asymmetrically reducing the large width, as was subsequently explained by Stupakov [61].

The optimized ID transition was installed in the APS storage ring and its impedance was measured by the local bump method [70,71], with the expectation that the measured vertical kick factor would be reduced by $\sim 30$. Unfortunately, it was observed that the "optimized" kick factor was about 20\% larger than that of the usual transition. Additionally, the aperture as measured by the electron beam was 1 to $2 \mathrm{~mm}$ smaller than expected. For this reason, the chamber was replaced.

After the optimized ID chamber was removed, subsequent measurements revealed an unusually large weld bead at both ends. The weld bead was located where the transition joined the narrow gap ID straight, and measured to protrude into the chamber by an average height $\sim 0.8 \mathrm{~mm}$. This discovery both explained the measured reduction in the aperture and would also lead to additional wakefields that were not accounted for in the initial analysis. To determine whether the weld beads could account for our wakefield measurements, we used GdfidL to compute the kick factor of the new transition including obstacles (weld beads) of varying heights. The results are plotted in Fig. 7(b), where we see that small obstacles whose height is $\sim 0.6 \mathrm{~mm}$ eliminate any impedance advantage of the optimized transition. Furthermore, we expect that the measured $0.8 \mathrm{~mm}$ weld beads will actually increase impedance. For comparison, Fig. 7(b) also includes the theory of small obstacles from Refs. [72-74], while we expect the predicted quadratic increase of the kick factor on height, the quantitative agreement is somewhat surprising since the theory is idealized.

The simulation results in Fig. 7(b) are for the transition alone, but comparisons to the experiment must also include the contribution due to the resistive wall of the $5 \mathrm{~m}$ long ID chamber. We plot the total change in the kick factor as a function of the obstacle height in Fig. 7(c), with theory in red and the measurement labeled by the blue arrow. Figure 7(c) shows that the best estimate of the measured weld bead height eliminates nearly all the discrepancy between the ideal optimal and the measurement, but a small difference remains. Regardless, these results show how significant imperfections and unintentional obstacles can be, particularly when they occur at narrow apertures. Indeed, for this reason the transitions to and from the IDs in the APS-U have been designed to be cut directly from the ID straight extrusion, thereby ensuring a smooth surface at the small gap.

The second example is based upon numerical simulations of possible bellows designs for the electron-ion collider (EIC) project [75]. The EIC will have a highintensity electron beam of 2.5 A with a $\sim 7 \mathrm{~mm}$ bunch length [76], and one considered bellows solution adapts the comb-type bellows that was originally developed in the High Energy Accelerator Research Organization (KEK) for high-current accelerators $[77,78]$. We chose to start with the design optimized by the Sirius project [79] shown in Fig. 8 (a), in which a flexible rf contact spring (circled in red) was introduced to eliminate higher order modes below $1 \mathrm{GHz}$ by eliminating the capacitive gap between adjacent fingers. This design was then modified to fit the EIC's $80 \mathrm{~mm}$ by $36 \mathrm{~mm}$ full-gap elliptical chamber, with the result shown in Fig. 8(b).

The impedance of the comb-type bellows will depend in part upon the length $L$ that the fingers overlap and the gap $g$ 


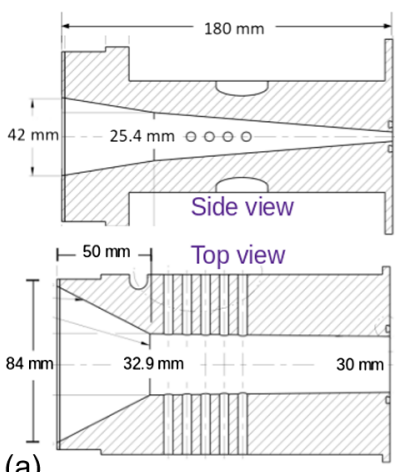

(a)

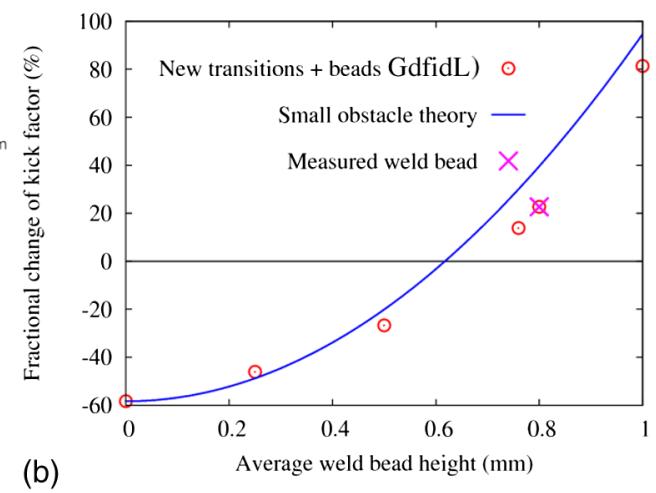

(b)

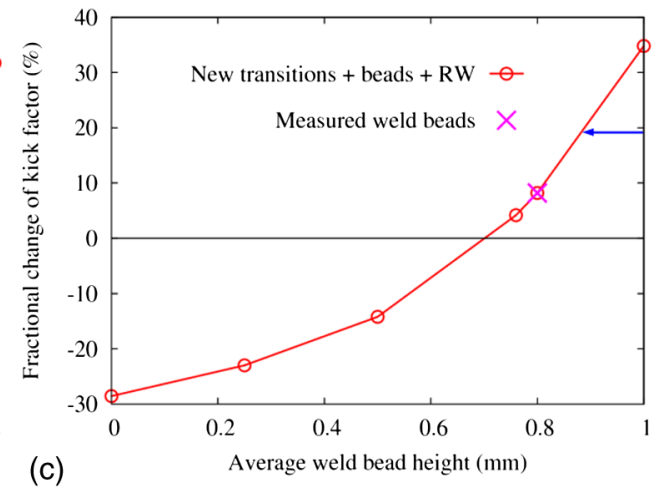

FIG. 7. (a) Geometry of the optimized transition. (b) Simulated change in the kick factor of the optimized transitions from that of the original. The solid blue line is from the theory of small obstacles. (c) Simulated change in the kick factor of the optimized ID including the resistive wall as a function of the weld beam height. The beam-based measurement is indicated by the blue arrow.

between the finger tip and the bellows body [see Fig. 8(c)], and these dimensions will change as the bellows is compressed or decompressed. Since the level of compression will vary around the ring depending upon available space and tolerances, we performed electromagnetic simulations to determine how the impedance varies with $g$ and $L$. We show in Figs. 9(a) and (b) that stretching the bellows $4 \mathrm{~mm}$ from its nominal dimensions of $g=20$ and $L=8 \mathrm{~mm}$ (orange lines) to $g=24$ and $L=4 \mathrm{~mm}$ (green lines) significantly changes the real part of the longitudinal impedance below $20 \mathrm{GHz}$. The appearance of the prominent mode near $13 \mathrm{GHz}$ in the stretched bellows (green) results in nearly three times the loss factor when the bunch length $\sigma_{s}<6 \mathrm{~mm}$. Hence, the level of rf heating can be expected to vary by a similar amount as the bellows geometry changes.

We also compare these two results with a different comb design that has the gap $g$ reduced to $6 \mathrm{~mm}$. Figure 9(c) shows that loss factor for this design is very similar to that with the same finger overlap length $L=8 \mathrm{~mm}$ when the bunch length is less than the gap length. For longer bunch lengths $\sigma_{s}>g$ the electromagnetic fields are effectively shielded such that they cannot penetrate through the slot.
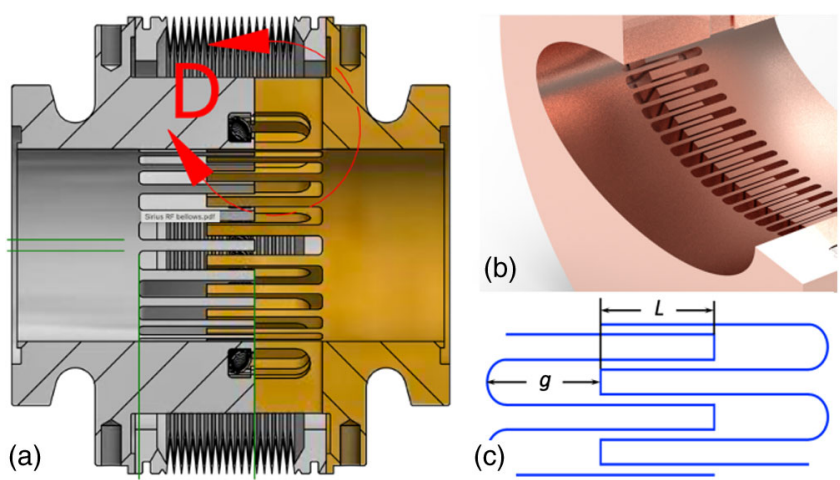

FIG. 8. (a) Sirius design of the comb-type bellows. (b) Combtype bellows as adapted for the EIC project with (c) the dimensions of the finger overlap $L$ and gap $g$.
The variations of the impedance shown here can potentially be eliminated by adapting a more conventional bellows design with rf fingers. Such a design can also reduce the impedance by nearly a factor of two, and therefore looks quite promising from an impedance perspective if a suitable water-cooling solution can be found.

\section{Interference effects between components}

The assumption that the wakefields from each component can be separately calculated applies when the geometric changes are small or the components are spaced far apart. These conditions typically apply in the arcs of most rings, typically fail for a series of rf cavities right next to each other, and may need to be carefully considered in other cases. Here, we contrast the situation of two transitions close together where interference effects can be important with that of a BPM-bellows assembly, where they can be safely ignored.

The NSLS-II has one straight section populated by two in-vacuum undulators (IVUs) that are close enough such that wakefield interference effects can be observed. We have found that this interference can lead to significant impedance contributions above the cutoff frequency. Unfortunately, detailed simulations of these 6-9 m long structures is computationally demanding, and in 3D may exceed what one can reasonably achieve. Here, we will illustrate the interference effect using a simplified 2D collimator geometry that can be simulated fairly quickly with the ECHO code [32].

Our simplified model of two back-to-back IVU's assumes axial symmetry and is sketched in Fig. 10. The basic structure involves a tapered transition from the maximum radius of $b_{\max }=12.5 \mathrm{~mm}$ to the minimum radius $b_{\min }=2.5 \mathrm{~mm}$ over a distance of $L_{\text {Tap }}=180 \mathrm{~mm}$. The length at the minimum aperture $g=500 \mathrm{~mm}$ is six times shorter than the real, $3 \mathrm{~m}$ magnet length of each IVU; this was chosen to ease the computational requirements but does not have a big impact on results. We will compare the impedance predictions when the transitions are $500 \mathrm{~mm}$ apart as shown in 


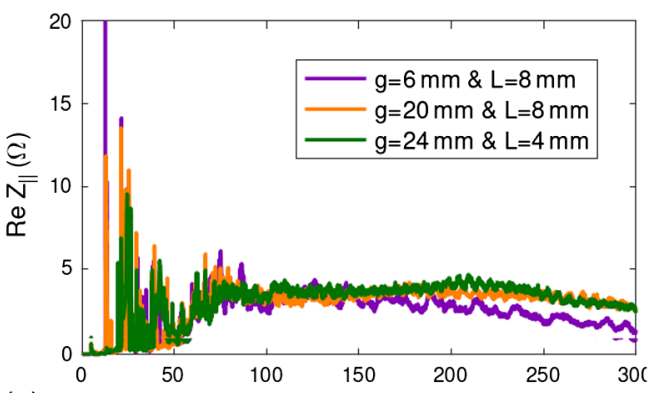

(a)

Frequency $(\mathrm{GHz})$

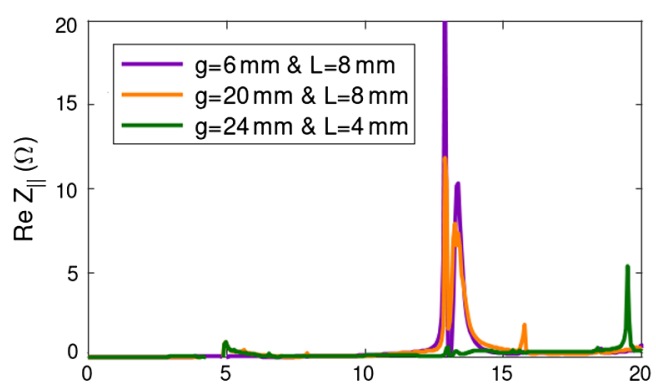

(b)

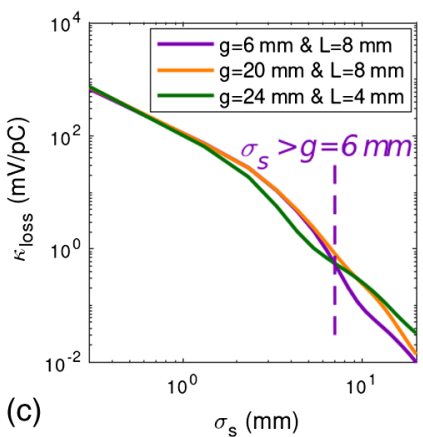

FIG. 9. (a) Real part of the impedance for three different sets of finger dimensions for the comb-type bellows. (b) Real part of the impedance below $20 \mathrm{GHz}$. (c) Loss factor as a function of bunch length.

Fig. 10 with the case where we simulate a single transitioned structure and multiply by two.

The results for the computed longitudinal impedance are presented in Fig. 11, where the red trace uses a single tapered collimator, the cyan trace multiplies the single tapered collimator result by 2 , and the dark purple trace is for two back-to-back tapered collimators. The average impedance behavior of the back-to-back collimators is very close to that of twice the single collimator. In particular, the two have essentially identical $\Im\left(Z_{\|} / n\right)=$ $\Im\left(Z_{\|}\right) /\left(\omega / \omega_{0}\right) \approx 0.28 \mathrm{~m} \Omega$ for $\omega \rightarrow 0$, and both agree reasonably well with Yokoya's analytic result for an axially symmetric taper [80],

$$
\Im\left(Z_{\|}\right) / n=\frac{\omega_{0} Z_{0}}{4 \pi c} \frac{\left(b_{\max }-b_{\min }\right)^{2}}{L_{\text {Tap }}} ;
$$

Using the NSLS-II's $\omega_{0}=2 \pi \times 378.5 \mathrm{kHz}$ and multiplying by two yields a theoretical prediction of $\Im\left(Z_{\|}\right) / n \approx$ $0.265 \mathrm{~m} \Omega$.

While the general behavior of the impedance is similar, having two back-to-back collimators creates a cavitylike structure between them. This in turn results in the appearance of many higher order modes above the cutoff frequency of $f_{c} \approx 2.4 c / 2 \pi b_{\max }=9.2 \mathrm{GHz}$ for the lowest longitudinal $E_{01}$-mode like in a circular waveguide. The forest of modes above $9 \mathrm{GHz}$ can be clearly seen by the purple lines of Fig. 11.

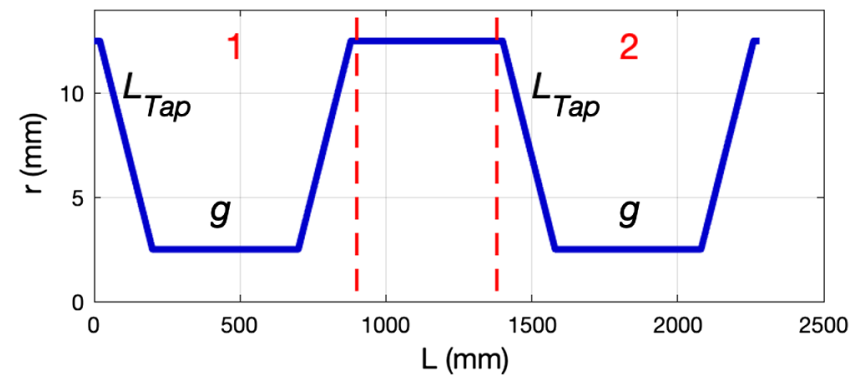

FIG. 10. Two back-to-back axially symmetric collimators separated by a $500 \mathrm{~mm}$ distance.
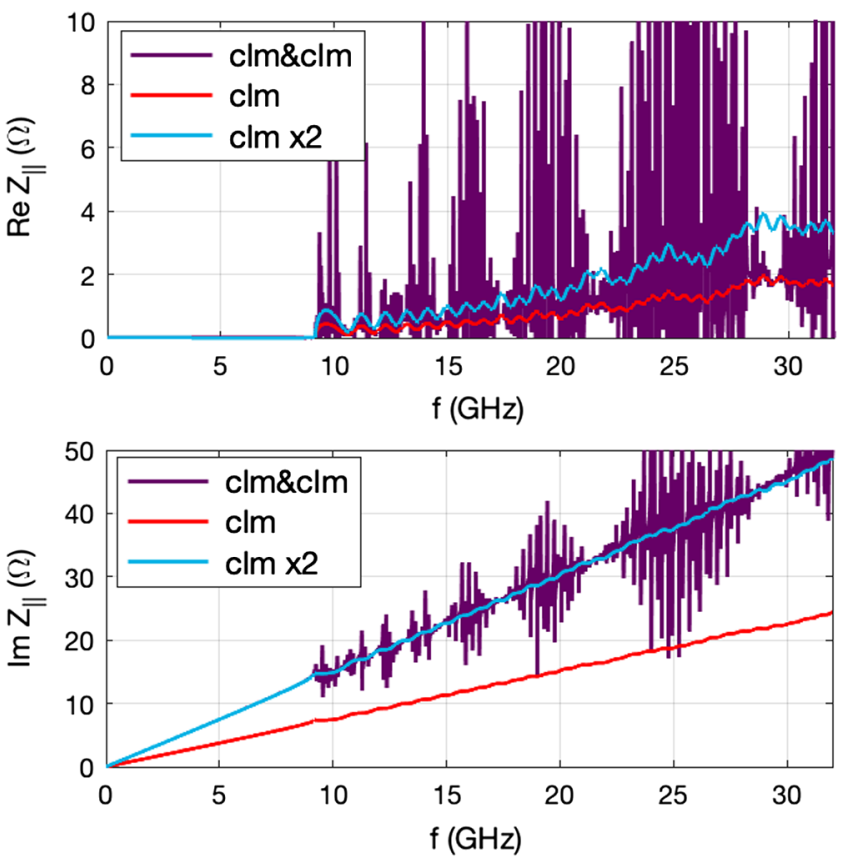

FIG. 11. The real (top) and imaginary (bottom) part of the longitudinal impedance up to $32 \mathrm{GHz}$ frequency range for the axially symmetric collimators.

Figure 12 compares the transverse impedance for the same three cases. In this case, the cutoff frequency is defined by the lowest vertical $H_{11}$-mode, which has $f_{c} \approx 1.84 c / 2 \pi b_{\max } \approx 7 \mathrm{GHz}$. While again the back-toback case has a very different higher order mode structure, at frequencies below $7 \mathrm{GHz}$ its impedance is indistinguishable from that of a single collimator multiplied by two. The simulated $Z_{\perp}(\omega \rightarrow 0) \approx 4.2 i \mathrm{k} \Omega / \mathrm{m}$ for two back-to-back collimators is twice that of a single collimator, and furthermore agrees reasonably well with Yokoya's calculation for the low-frequency transverse impedance [80,81]:

$$
Z_{\perp}(\omega \rightarrow 0)=\frac{i Z_{0}}{4 \pi} \frac{\left(b_{\max }-b_{\min }\right)^{2}}{L_{\text {Tap }} b_{\min }^{2}} \approx 2.7 i \mathrm{k} \Omega / \mathrm{m}
$$

for our geometry. 

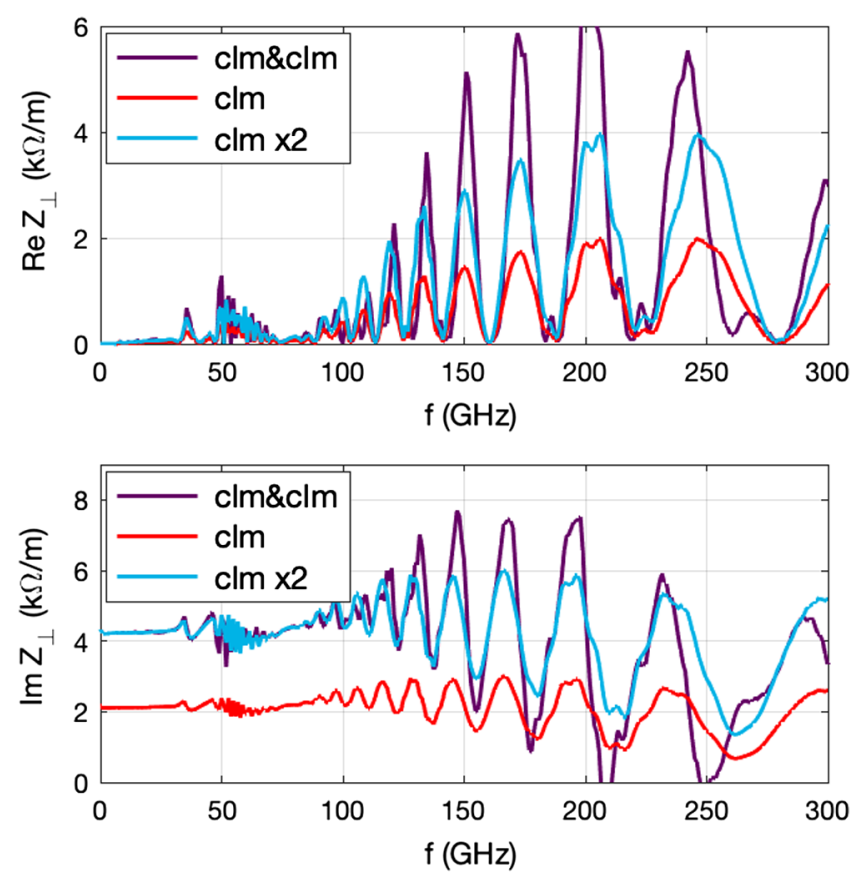

FIG. 12. The real (top) and imaginary (bottom) part of the transverse impedance up to $300 \mathrm{GHz}$ frequency range for the axially symmetric collimators. $\operatorname{Im} Z_{\perp}(\omega \rightarrow 0)=2.1 \mathrm{k} \Omega / \mathrm{m}$ per collimator.

The previous example showed that the introduction of a cavitylike structure between components can lead to an impedance that is not simply the sum of the two contributions. In a similar manner, structures that are closely spaced and periodic may have important interference effects. Extreme examples of such situations include sequences of $\mathrm{rf}$ cavities or specially designed corrugated structures. Nevertheless, we have found that the wakefield interference effects are negligible for most situations that arise in storage ring.

As an extreme example of a situation where interference effects are small, we consider the APS-U bellows/BPM assembly that will be discussed further in Sec. V and is shown in Fig. 15. Here, we first separately simulated the green, central region to assess the impedance characteristic of the BPM buttons shown in gray. We then eliminated the buttons from the model, and added the rf contact fingers and compression springs to verify that the fingers provided good rf shielding and that the small cavity formed by the bellows was acceptable. The resulting wakefields are shown in Fig. 13(a). In Fig. 13(b), we compare the wakefield sum of the two contributions from (a) to $W_{\|}(s)$ from the full bellows/BPM assembly, showing that the two predictions agree quite well.

Figure 13(b) indicates that there are cases where the interference effects within even a single component are small. For the bellows/BPM assembly here the two solutions with different boundary conditions approximately sum because each is a small perturbation of the other.
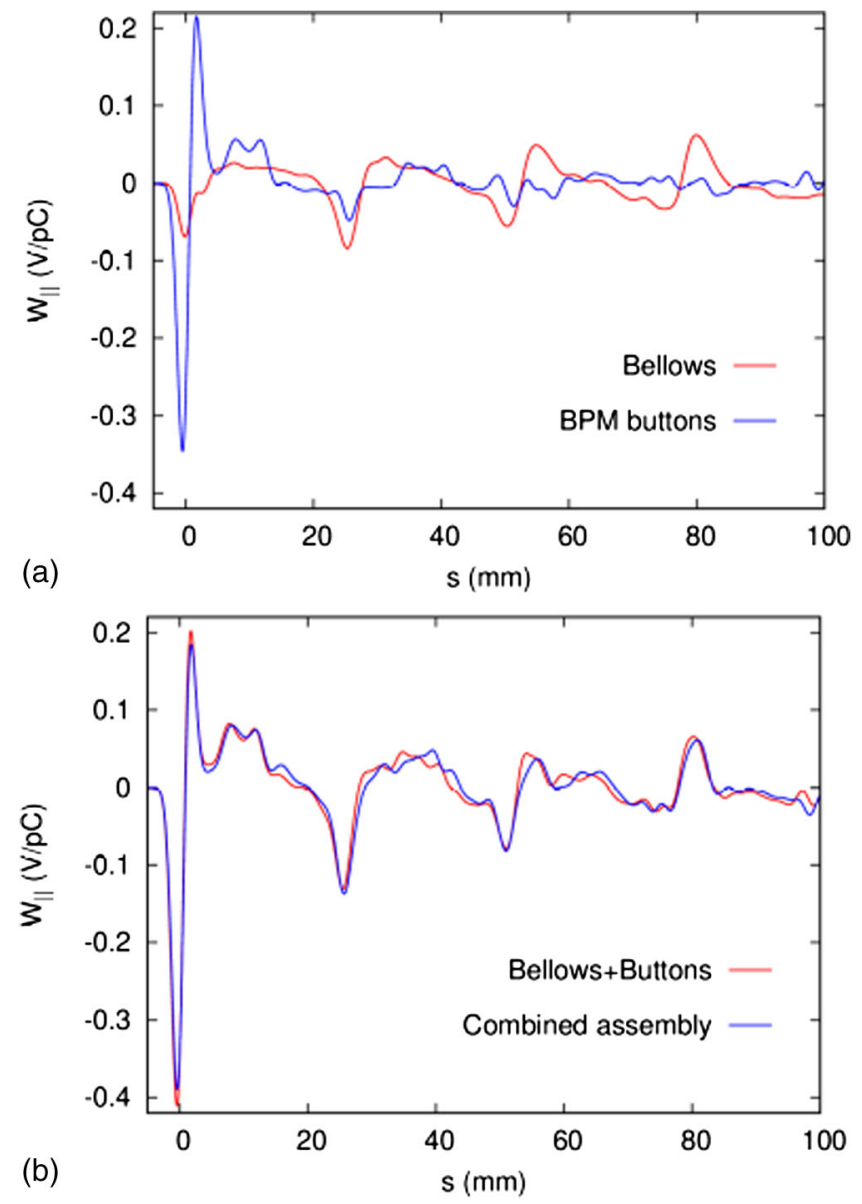

FIG. 13. (a) Wakefields from simulations that separately focused on the APS-U bellows and BPM buttons shown in Fig. 15. (b) Comparison of the sum between the two contributions from (a) to that of the entire structure.

Similarly, we have found that interference effects between different components can be neglected if each structure represents a small perturbation (meaning that its impedance is small), and furthermore that the structures do not form a periodic array with strong resonantlike effects. Hence, the usual procedure to individually analyze components is typically valid.

\section{DATA POST-PROCESSING}

Electromagnetic codes typically output the wakefield as a function of distance $s$ behind the peak of a Gaussian electron bunch. Once this has been computed for various components, the data can be analyzed and processed for subsequent simulations. This analysis can be done with a number of tools including MATLAB, Mathematica, or Python scripts, and usually includes plots of the longitudinal and transverse wakefields $W_{\|, \perp}(s)$, the real and imaginary parts of the associated impedances $Z_{\|, \perp}(k)$, and several other processed quantities. For example, the wakefield for the circulating bunch length $\sigma_{s 0}$ can be found 


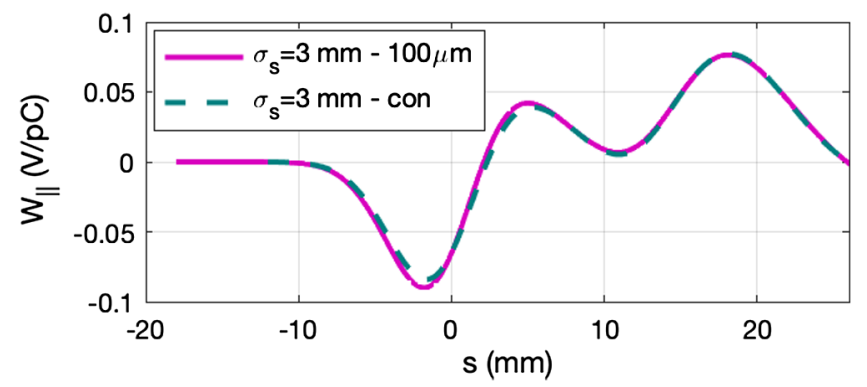

FIG. 14. Wakefield comparison for the NSLS-II flange absorber. The pink trace is the longitudinal wakefield simulated directly for a $3 \mathrm{~mm}$ bunch length. The dashed dark-cyan trace is a pseudo-Green's function computed for a $\bar{\sigma}_{s} \sim 0.3 \mathrm{~mm}$ bunch length and then convolved with a $3 \mathrm{~mm}$ Gaussian bunch, which agrees with the direct calculation.

by the following convolution with the pseudo-Green's function:

$W\left(s, \sigma_{s 0}\right)=\int d s^{\prime} \bar{W}\left(s-s^{\prime}, \bar{\sigma}_{s}\right) \frac{\exp \left[-\frac{\left(s-s^{\prime}\right)^{2}}{2\left(\sigma_{s 0}^{2}-\bar{\sigma}_{s}^{2}\right)}\right]}{\sqrt{2 \pi\left(\sigma_{s 0}^{2}-\bar{\sigma}_{s}^{2}\right)}}$.

Figure 14 plots the wakefields computed for the NSLS-II flange absorber presented in Fig. 3. The dashed dark-cyan trace is the pseudo-Green's function computed for a $\bar{\sigma}_{s} \sim$ $0.3 \mathrm{~mm}$ bunch length and convolved with a $3 \mathrm{~mm}$ Gaussian bunch. The resulting wakefield agrees perfectly with the pink trace, which is the simulated wakefield from a $3 \mathrm{~mm}$ bunch with a stepsize $100 \mu \mathrm{m}$.

For the beam-induced heating and coupled bunch instability analysis, the wakefield can be computed using a test bunch whose length equals that circulating in the ring. In this case, $W_{\|}(s)$ can be computed with an increased stepsize $(\Delta \sim 100 \mu \mathrm{m})$, which allows for wakefields computed over a much longer distance $s$. Since these long-range wakefields result in high-resolution impedances, they can be used to compute electrodynamics parameters such as the shunt impedance $\left(R_{\mathrm{sh}}\right)$ and the quality factor $(Q)$ that are required for coupled-bunch instability analysis. In addition, the loss factor and the kick factors that are used for beaminduced heating and for the transverse instability threshold estimations can also be computed from wakefields obtained from the natural bunch length. We will discuss the loss factor further in Sec. VI.

As an example, we now discuss how such analysis was applied to the APS-U bellows/BPM assembly shown at the top of Fig. 15. For this geometry, the radius of the standard vacuum chamber aperture is $11 \mathrm{~mm}$, the BPM button diameter is $8 \mathrm{~mm}$, and the downstream and upstream rf contact fingers are compressed against the central BPM body pipe that is green in Fig. 15. The simulated real part of the impedance $\Re Z_{\|}(f)$ is shown in the middle plot. The impedance is derived from the longitudinal wakefield of a $5 \mathrm{~mm}$ bunch length using $Z_{\|}(k)=\mathrm{FT}\left[W_{\|}(z)\right] / \mathrm{FT}[-c Q(z)]$,
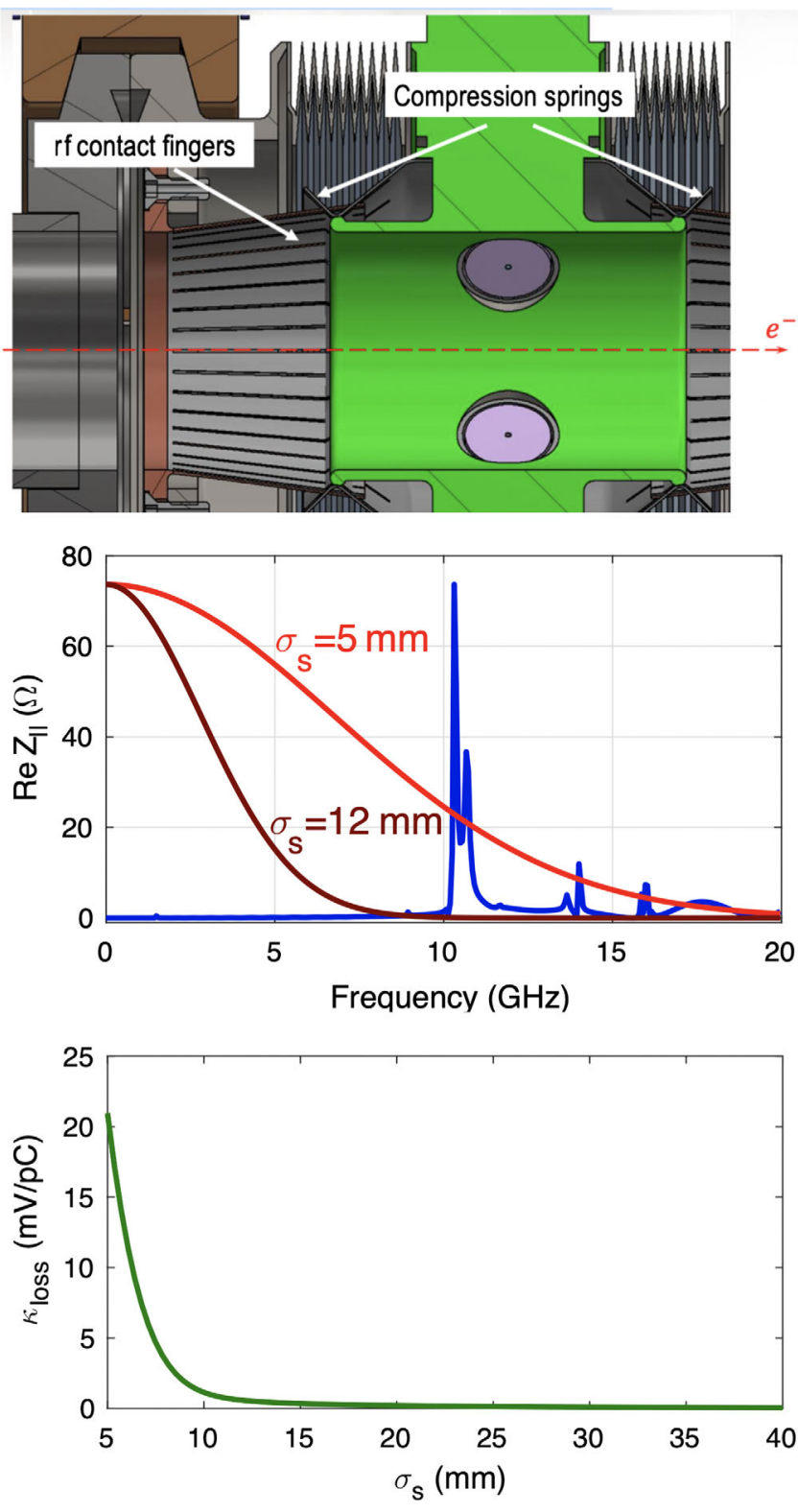

FIG. 15. Top: internal view of the APS-U Bellows/BPM assembly. Middle: plot of the real part of the longitudinal impedance (blue trace), the Gaussian spectrum for the $5 \mathrm{~mm}$ bunch length used to compute $W_{\|}$(red), and the spectrum from a $12 \mathrm{~mm}$ bunch (purple). Bottom: The loss factor vs the bunch length for the APS-U bellows/BPM assembly.

where, e.g., $\mathrm{FT}[Q(z)]$ is the Fourier transform of the charge profile. The first resonance is observed at $\approx 11 \mathrm{GHz}$, which could result in significant heating if the bunch length was $5 \mathrm{~mm}$ (spectrum in red). The APS-U, however, plans to lengthen the bunch to $30 \mathrm{~mm}$ to increase lifetime, and we show that even a $12 \mathrm{~mm}$ bunch has a small spectral overlap with the impedance, and correspondingly small heating. This dependence is then summarized at the bottom of Fig. 15, where we plot the loss factor as a function of bunch length.

Once the wakefields for each individual component have been calculated, separate scripts can be used to combine 


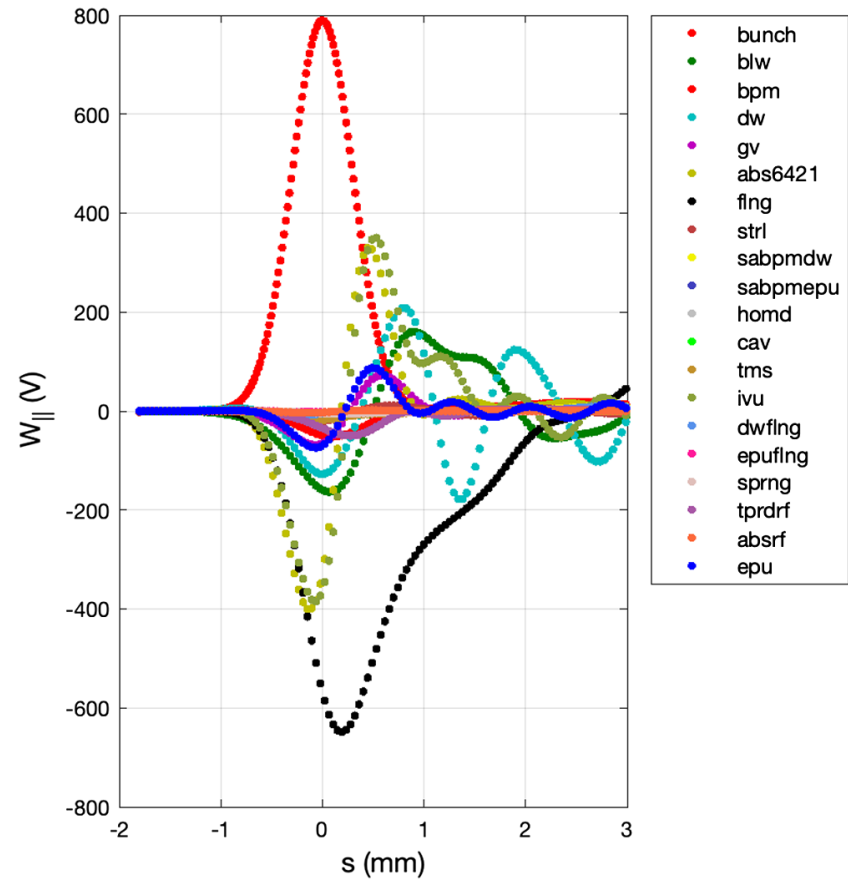

FIG. 16. The NSLS-II longitudinal short-range wakefields calculated for a $0.3 \mathrm{~mm}$ bunch length for each individual component.

them into a total wakefield that can be used for further tracking simulations of collective effects. Figure 16 shows all the geometric contributions to the longitudinal wakefield of the NSLS-II [82], which are summed into the total wakefield plotted in Fig. 17. The first largest sources contributing to the longitudinal wakefields are the $739 \mathrm{rf}$ shielded flanges, more than 100 flange absorbers, and nine small gap IVUs. Finally, the Fourier transform of the total, ring-averaged wakefield yields the total impedance shown in Fig. 18. Here, we also show how different the predicted impedance can be if one misses an important component. While the impedance of any single rf-shield flange is relatively small, the large number of such flanges leads to a critical contribution to the total impedance.

The simulation and modeling techniques described here that compute wakefields and their effects on the beam typically agree quite well with experiments measuring single components. Nevertheless, the predictions made for an entire facility are often less accurate. Many groups, including SOLEIL, RHIC, Super-KEKB, DIAMOND, and the NSLS-II have observed discrepancies by a factor of 2 or more between numerical predictions and experimental measurements in terms of instability thresholds and other collective effects [83]. There has been much discussion to try to understand why predictions based upon the entire impedance model often underestimate collective effects. One possible explanation is that the interference effects described earlier play an important role; another is that the impedance model has either neglected relevant sources of impedance, or simulated idealized models that differ

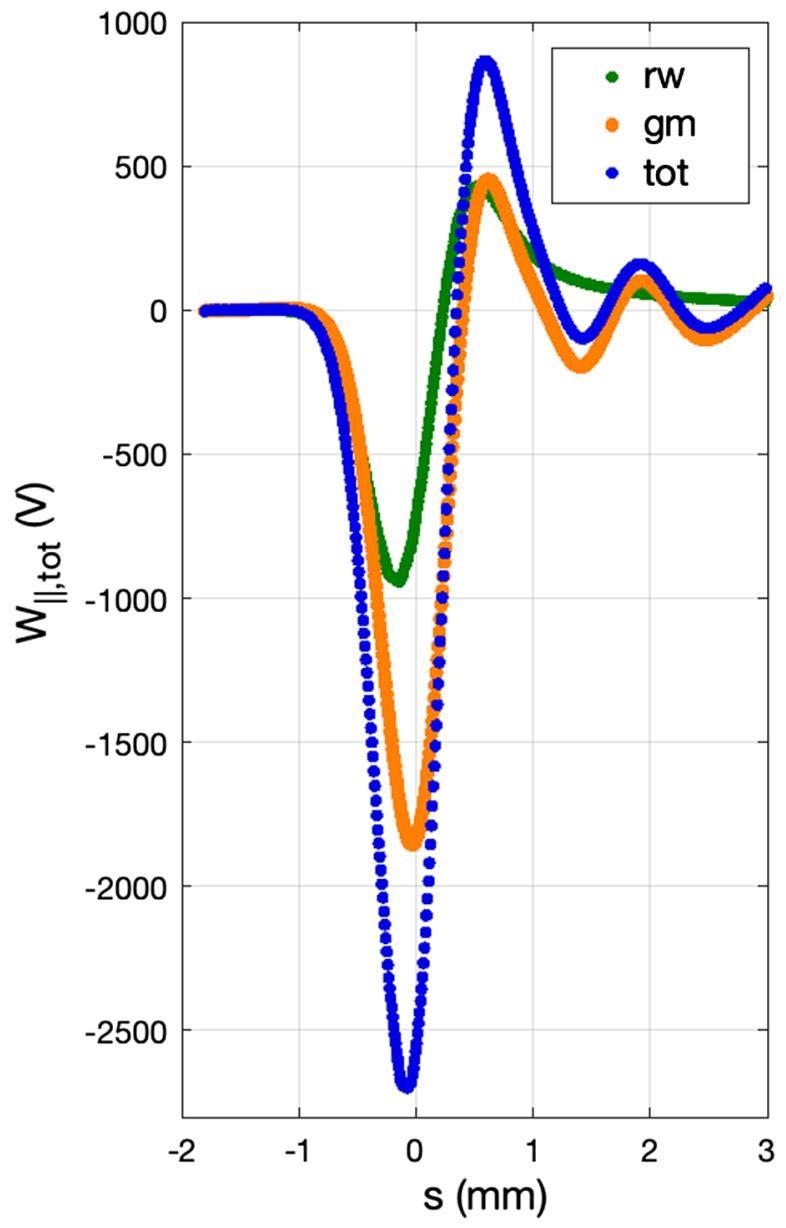

FIG. 17. The total longitudinal wakefield of the NSLS-II storage ring (blue trace) as a sum of the resistive wall contribution (green trace) calculated analytically and the geometric wakefields (orange trace).

somewhat from the installed components. Adding up even small differences over an entire ring may lead to quite different predictions. Better understanding of the source(s) of the observed discrepancies would be a significant achievement, since it will help improve predictions for future upgrade projects and reduce the risk that ring performance will be unexpectedly limited by collective effects.

\section{BEAM-INDUCED HEATING}

The beam-induced heating is of significant concern at many high-current accelerator facilities. Localized overheating of vacuum components can limit both the single-bunch current and the total average current during the commissioning phase and regular beam operation. To avoid the disaster of vacuum component deformation, damage and/or failure due to the image-current heating, temperature transfer analysis should be performed for sensitive vacuum components before their installation into the ring. While equations for purely resistive heating are available, there is at present no 

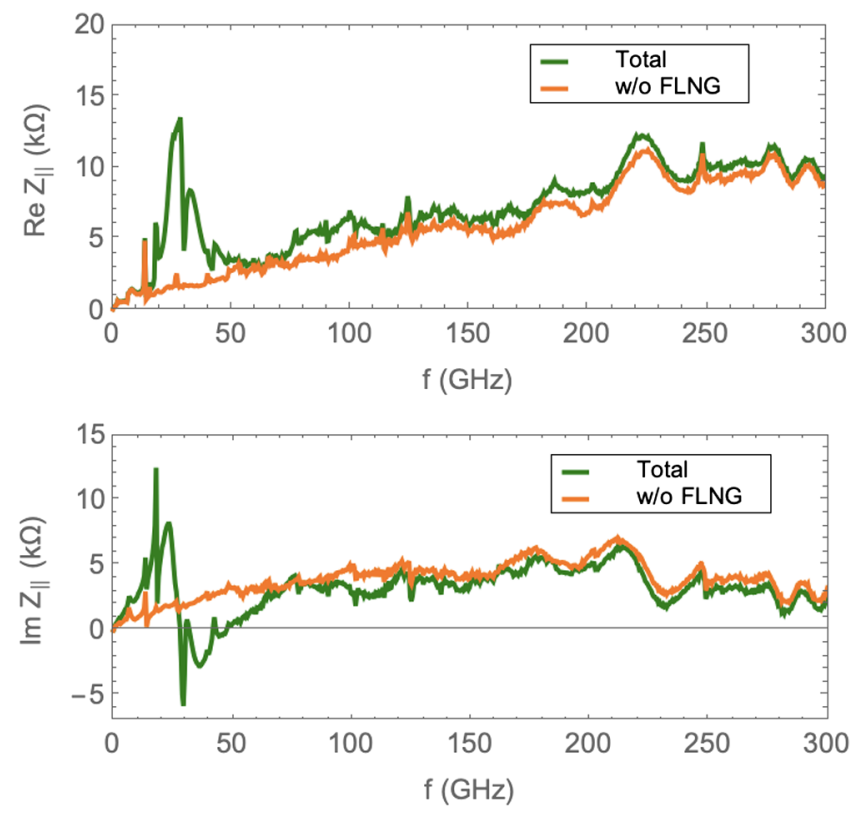

FIG. 18. The real (top) and imaginary (bottom) part of the total longitudinal impedance up to $300 \mathrm{GHz}$ frequency range. Green and orange traces are with and without contribution of the $\mathrm{rf}$ shielded flanges respectively.

direct method to analytically calculate the beam-induced heating for accelerator components. Rather, one typically begins with the expression for the parasitic power loss [84]

$$
P_{\text {loss }}=-\Delta E / T_{0},
$$

where $T_{0}$ is the revolution period and $\Delta E$ is the energy loss of the beam into the component under consideration.

The energy loss for a single bunch in a storage ring is proportional to the product of the periodic beam spectrum with the impedance, being given by [84]

$$
\Delta E=-\left(e N_{p}\right)^{2} \frac{\omega_{0}}{2 \pi} \sum_{n=-\infty}^{\infty}\left|\tilde{\rho}\left(n \omega_{0}\right)\right|^{2} \Re\left[Z_{\|}\left(n \omega_{0}\right)\right]
$$

where $N_{p}$ is the number of particles in the bunch and $\tilde{\rho}$ is Fourier transform of the longitudinal profile normalized to unity. There is a resonant enhancement of energy loss if the impedance has a peak of width $\lesssim \omega_{0}$ centered at a harmonic of the revolution frequency $\omega_{0}$. While the heating associated with these resonances can in principle be avoided by small changes in component geometry to shift the resonant frequency of the impedance, in general it is best to try to design structures without such sharp resonances. In the case where the impedance varies slowly over the frequency scales $\sim \omega_{0}$, then the sum can be approximated as an integral, and the power loss for $M$ bunches reduces to

$$
P_{\text {loss }}=\frac{\left(e N_{e}\right)^{2}}{T_{0}} \frac{M}{2 \pi} \int_{-\infty}^{\infty} d \omega|\tilde{\rho}(\omega)|^{2} \Re Z_{\|}(\omega),
$$

$$
=\frac{I_{\mathrm{ave}}^{2} T_{0}}{M} k_{\mathrm{loss}}
$$

where $I_{\text {ave }}=e N_{e} M / T_{0}$ is the average current and $k_{\text {loss }}$ is the loss factor.

The prefactor in Eq. (22) is determined by machine parameters, so from an impedance prespective the main quantity of interest is the loss factor. For a Gaussian bunch of length $\sigma_{s}$, the loss factor can be expressed as

$$
k_{\text {loss }}\left(\sigma_{s}\right)=\frac{c}{\pi} \int_{0}^{\infty} d k e^{-k^{2} \sigma_{s}^{2}} \Re\left[Z_{\|}(k)\right],
$$

where $Z_{\|}(k)$ is the impedance associated with a point charge. If the impedance is obtained as the Fourier transform of a wakefield simulated with the bunch length $\bar{\sigma}_{s}$, then we have

$$
k_{\mathrm{loss}}\left(\sigma_{s}\right)=\frac{c}{\pi} \int_{0}^{\infty} d k e^{-k^{2} \sigma_{s}^{2}} \Re\left[e^{\bar{\sigma}_{s}^{2} / 2} Z_{\|}\left(k, \bar{\sigma}_{s}\right)\right] .
$$

The quantity in brackets "deconvolves" the impedance with the source charge, and in some cases is what the wakefield solver will actually output.

The energy loss due to wakefields scales with the real part of the impedance and becomes more significant for shorter bunches that can interact with higher frequency components of the impedance. The power due to any component can therefore be computed once the total impedance, including both geometric and resistive components, is calculated.

While Eqs. (22) and (23) give the power lost by the beam, it unfortunately does not say where this energy goes. Some fraction of the power will be trapped and dissipated within the structure itself, while the rest may propagate down the pipe and be deposited elsewhere. Determining how much power is dissipated in what part of a given component can be computed using certain simulation codes including CST Microwave studio [30]. To convert this into component heating and actual temperature changes, however, still requires additional calculations with something like the ANSYS finite element analysis code [85]. Hence, making detailed predictions of the rf heating for all vacuum components can be a very computationally intensive program that requires significant time and effort.

One strategy to reduce the amount of analysis is to compute the loss factor using the natural bunch length $\sigma_{s 0}$ that does not include any bunch lengthening, and to apply the very conservative assumption that all the lost energy is deposited in the structure. If this worst-case level of rf heating is deemed manageable then no further analysis is required. On the other hand, if the power is too high then one must consider whether to proceed with further analysis, add cooling, or change the component design. Since the latter two options can result in significant engineering effort, 

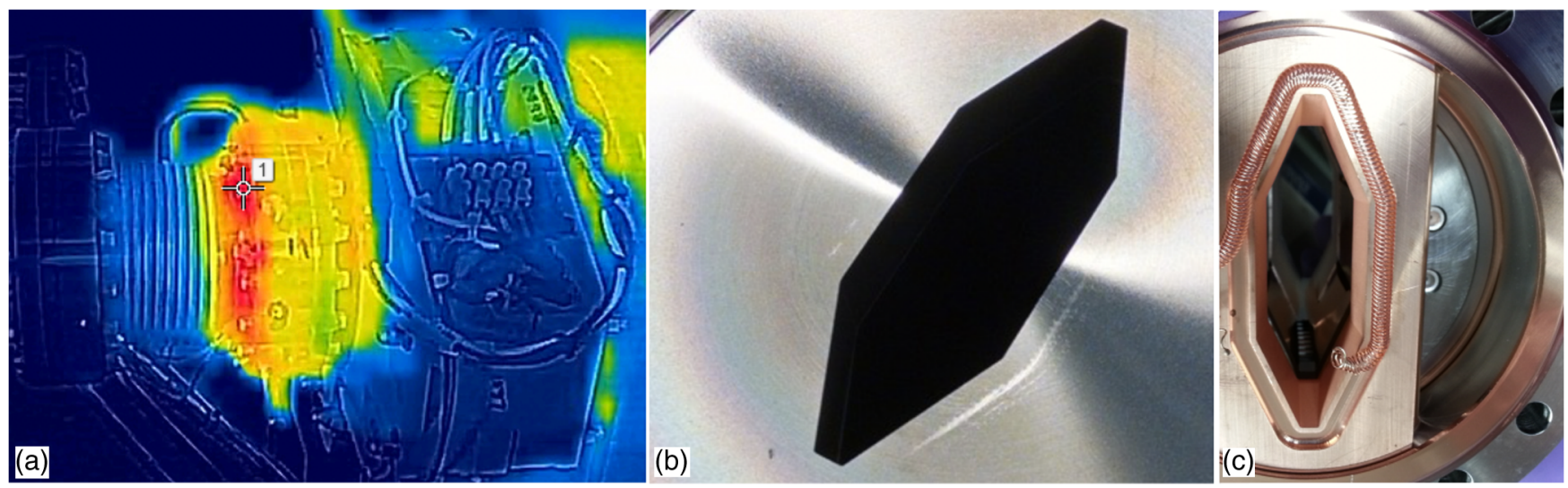

FIG. 19. (a) Thermal view of the NSLS-II Bellows with improperly installed the rf contact spring. (b) The SS flange surface has a scratch where the rf spring has good contact, but at least $50 \%$ of the area shows little evidence of this contact. (c) Image of the flexible rf spring which was displaced from the trapezoidal grove during installation, and as a result was damaged by synchrotron radiation.

design complexity, and/or cost, ideally some combination of analysis and component design should be used to balance heating margin with the costs of over-designing components.

Even after careful design, it is a good idea to monitor the temperature of sensitive components during operation. For example, the NSLS-II has continually monitored the temperature of various vacuum components using resistance temperature detectors. Temperature monitoring significantly reduced the risk of unexpected component failure and major vacuum leaks throughout the commissioning and operating phase as the NSLS-II sought to achieve its target $I_{\text {ave }}=500 \mathrm{~mA}$ average current.

If local heating is observed on an resistance temperature detectors one can then employ Infrared cameras to more clearly identify where the temperatures are highest. We show a thermal image from an IR camera in Fig. 19(a), which clearly indicates an elevated temperature in the flange joint on the right-hand side of the bellows. This joint was opened during a subsequent maintenance period, and it was found that the spring designed to ensure good rf contact was not properly installed. Figure 19(b) shows that the imprint of the spring contact on the adjoining flange only covered part of its surface, indicating poor rf contact between the spring and the flange. As shown in Fig. 19(c), this was because the spring was not properly set in its groove. The damage to the spring was from synchrotron radiation as it hung into the chamber.

\section{SIMULATING COLLECTIVE EFFECTS WITH TRACKING}

It is important to recognize that any attempt to model collective effects will only be as good as its ability to properly identify and model the various sources of impedance in the ring [86]. Since any change in the vacuum chamber geometry is a potential source of wakefields, identifying the "important" and/or "relevant" components is an art that relies on experience. Previously in the paper, we have mentioned components that typically must be accounted for, including BPMs, bellows, ID and rf transitions, flanges, gate valves, rf cavities, photon absorbers, collimators, scrapers, and stripline kickers. Once the components have been identified, electromagnetic solvers can be used to compute the associated wakefield and impedance of each component as we discussed earlier. Here, we will show how the fidelity of these simulations can impact tracking predictions of collective effects.

Once the impedance elements have been identified and simulated, they can be used to build an impedance model for tracking. The simplest such model lumps all sources of impedance into a single "impedance element" that is then applied once per turn. The longitudinal impedance within this model is determined by summing the impedance contribution from all components in the ring, $Z_{\|}^{\text {tot }}(\omega)=$ $\sum_{j} Z_{\|, j}(\omega)$ for each component $j$, while the total transverse impedance of the ring is found by weighting the individual geometric contributions by their respective local lattice functions and summing. More precisely, the total geometric impedances along $x$ and $y$ are given by

$$
\begin{aligned}
Z_{x}^{\mathrm{tot}}(\omega) & =\frac{1}{\beta_{x}} \sum_{\text {elements } j} \beta_{x, j} Z_{x, j}(\omega), \\
Z_{y}^{\mathrm{tot}}(\omega) & =\frac{1}{\beta_{y}} \sum_{\text {elements } j} \beta_{y, j} Z_{y, j}(\omega),
\end{aligned}
$$

where the lattice functions at element $j$ are denoted by $\beta_{x, j}$ and $\beta_{y, j}$, while $\beta_{x}$ and $\beta_{y}$ are the lattice functions at the location in the simulated ring where the impedance element is applied. The influence of collective effects can then be computed with tracking by inserting the lumped (total) impedance at a single location within a code.

The lumped impedance model has proven to be quite accurate when the collective response is built up over many turns, as is the case for the longitudinal dynamics and most 
transverse instabilities. On the other hand, it is not always sufficient to simulate nonequilibrium effects that might occur during the transients of particle injection or right after a kicker has fired [87]. Nevertheless, these cases can typically be treated using similar techniques if one divides the impedance into multiple impedance elements that are then distributed around the ring.

The particle tracking itself can use several models, ranging from linear maps that include synchrotron emission to full element-by-element tracking. The former are sufficient to model longitudinal collective effects, while adding chromatic variations and lowest-order nonlinearities is typically sufficient to predict transverse stability at equilibrium. Again, predicting transient effects when the beam is potentially far from the nominal orbit presents the most demanding simulations, often requiring high-order maps or full element-by-element tracking.

Having outlined the basic methodology, we would like to discuss some specific details that are important to consider to obtain reliable predictions. We will illustrate these points with the impedance model developed for the APS-U. The first thing to recognize is that the most important part of any impedance model is identifying all the relevant impedance contributions. While perhaps obvious, it bears repeating since accounting for all of the necessary components is perhaps the least systematic step in the process. We illustrate this in Fig. 20, where we show what happens if we were to forget or miss the contributions of the flange gaps, kicker chambers, and the SS chambers. In this case, the $\Im\left(Z_{\|}\right) / n$ decreases by about $25 \%$, and the predicted level of bunch lengthening due to the impedance decreases by a similar amount. Interestingly, the observed microwave instability threshold is essentially unchanged. We will explain the reason for this shortly.

In addition to identifying all the components, one must also decide how to use the simulated wakefields in the tracking. This is complicated by the fact that the wakefield calculated from a finite difference solver is computed from a (typically Gaussian) charge distribution with finite extent, while the wakefield from an ultrarelativistic particle is causal in the sense that it only acts on trailing particles. Several methods have been developed to deal with this mismatch. For example, one can deconvolve the impedance with the source Gaussian, but this is in general numerically unstable and therefore only works over a limited frequency range. Alternatively, one can "reconstruct" the point-charge wakefield as described in Ref. [88]. This can be quite useful for short bunches whose length $\lesssim 0.1 \mathrm{~mm}$, but is numerically costly for the bunch lengths typically found storage rings. Another method involves fitting the impedance to a number of broadband resonators [89], but we have found this challenging to do in a general fashion since it requires specifying the unknown high-frequency behavior. Furthermore, any similar method leads to fine-structured wakefields (high-frequency impedance components) that require an extremely large number of particles to reliably simulate.

Since we do not expect that the collective behavior will depend on the behavior of the wakefield/impedance at very small length scales/very high frequencies, our approach has been to simply accept the wakefields as simulated. Then, if our electromagnetic code derived the wakefield using a bunch of length $\bar{\sigma}_{s}$, the numerical wakefields used in tracking are understood to be the point-charge wakefield that has been smoothed by a Gaussian filter of frequency width $c / \bar{\sigma}_{s}$. The only remaining item is to determine the required length $\bar{\sigma}_{s}$, which effectively means finding the frequency range over which the impedance affects the dynamics.

We have found that obtaining consistent predictions regarding the microwave instability require the bunch length $\bar{\sigma}_{s}$ to be sufficiently short such that it fully resolves the first large resonator-like peak of the impedance. We admit that this requirement is only loosely defined, and so we illustrate its use with an example. The APS-U longitudinal impedance shown in Fig. 21(a) has its first prominent resonance located near the cutoff frequency of the $11 \mathrm{~mm}$ radius beam pipe. This "resonance" is quite broad and due to several components including the BPMs and the in-line absorbers, while the sharper resonance near $20 \mathrm{GHz}$ is from the BPMs. We expect
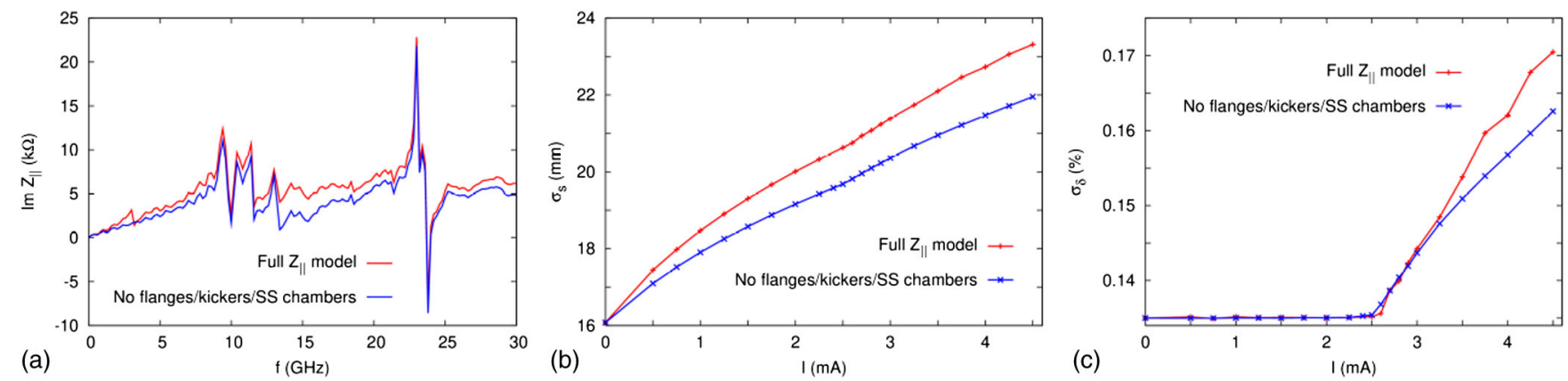

FIG. 20. (a) Imaginary longitudinal impedance from the full model (red) and where the flanges, kickers, and SS chambers have been missed (blue). (b) shows that the bunch lengthening changes by $20 \%-30 \%$, while (c) indicates that the microwave instability threshold is approximately unchanged. 

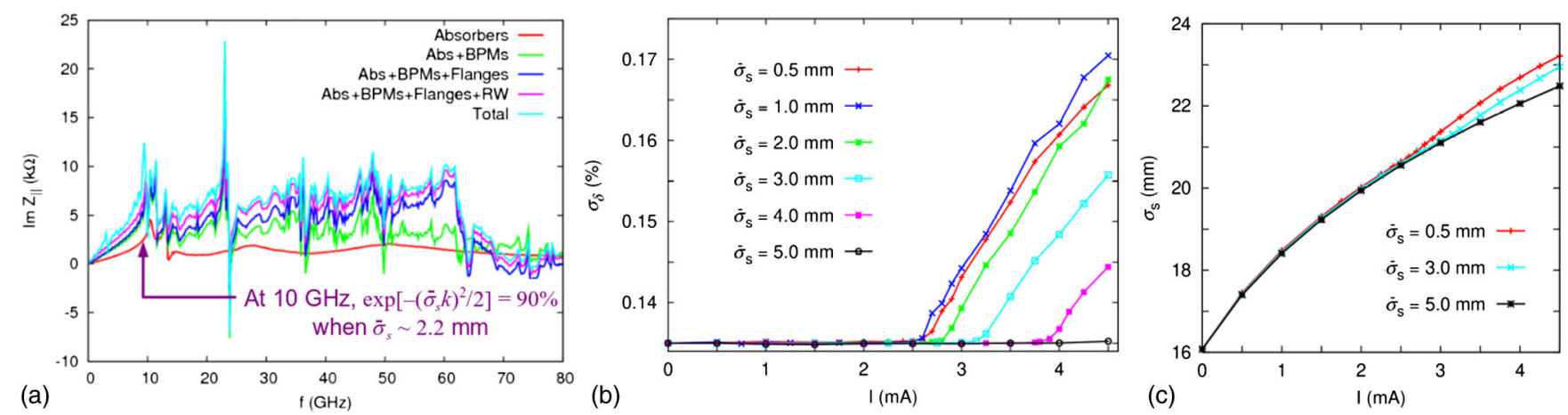

FIG. 21. (a) Computed longitudinal impedance of the APS-U. (b) Onset of the microwave instability for various frequency ranges of the impedance as defined by the wakefield solver bunch length $\bar{\sigma}_{s}$ consistent results requires resolving the first resonatorlike peak of the impedance. (c) The predicted bunch lengthening is nearly identical below the onset of the microwave instability.

that resolving the peak at $10 \mathrm{GHz}$ will be required to accurately predict the microwave instability threshold, and that the one at $20 \mathrm{GHz}$ may be important as well. Hence, we conclude that the wakefield should be calculated with a bunch length $3 \bar{\sigma}_{s} \lesssim c /\left(2 \pi f_{\text {res }}\right) \approx 4.8 \mathrm{~mm}$.

We illustrate how the frequency range of the impedance affects predictions in Fig. 21(b), which plots the predicted energy spread from tracking simulations as a function of the current for various values of $\bar{\sigma}_{s}$. This plot shows that the microwave instability threshold is significantly underestimated if $\bar{\sigma}_{s} \gtrsim 2 \mathrm{~mm}$ when the spectral range of the impedance is insufficiently small. On the other hand, when $\bar{\sigma}_{s} \lesssim 1 \mathrm{~mm}$ the first resonance peak is clearly resolved and we obtain consistent predictions. Note that the required wakefield solver bunch length $\bar{\sigma}_{s} \lesssim 1 \mathrm{~mm}$ is more then 15 times shorter than the electron bunch length in the ring, which we see from Fig. 21(c) is $>15 \mathrm{~mm}$.

Our observation that one must properly resolve the first resonator-like peak in order to accurately predict the microwave instability helps explain why the microwave threshold is essentially unaffected in Fig. 20 even as we reduce the impedance-while eliminating the flange gaps reduced the $\Im\left(Z_{\|}\right) / n$ and therefore the bunch lengthening, the position and prominence of the first "resonance" was relatively unaffected. Hence, the microwave instability threshold was largely unchanged. Finally, we note that obtaining consistent simulations requires a sufficient number of macroparticles per frequency bin, so that more particles are needed as one decreases $\bar{\sigma}_{s}$. We typically sample the impedance at a frequency spacing $\sim 0.2 \mathrm{GHz}$ and use between 10000 and 800000 particles per bunch, depending on the application. Predicting the microwave instability tends to be the most demanding, and we find that $\gtrsim 1000$ particles/frequency bin is typically required.

In order to simulate transverse collective effects properly, one first needs to satisfy all of the previous requirements. In particular, one must find all the relevant sources of impedance, use a sufficient number of particles, and have a suitable frequency range of the impedance so that the longitudinal collective effects are correct. Getting the longitudinal dynamics properly modeled is crucial both because the transverse kick is obtained from a convolution of the current profile and the dipole wakefield, and because transverse stability depends upon the energy spread through chromatic effects. Hence, one should not expect reasonable transverse predictions without first obtaining the correct longitudinal phase space including wakefields.

In addition to the requirement for having the correct longitudinal dynamics, one also needs to ensure that the transverse impedance can resolve both the current shape and the chromatic frequency shift $\omega_{\xi}=\xi \omega_{0} / \alpha_{c}$, where $\xi$ is the chromaticity and $\alpha_{c}$ is the momentum compaction. The chromatic frequency shift reflects the fact that the betatron frequency varies with energy, which in turn results in the phase accumulation of

$\omega_{\xi} s / c=\int d \tau \frac{\Delta \omega_{\beta}}{c}=\int d \tau \frac{\omega_{0} \xi \delta}{c}=\int d \tau \frac{\omega_{0} \xi}{c \alpha_{c}} \frac{d s}{d \tau}$,

as the particle undergoes synchrotron oscillations [90].

Typically, simulations will resolve the frequency $\omega_{\xi}$ if the bunch length $\bar{\sigma}_{s}$ is short enough to properly model the longitudinal collective effects. Nevertheless, we illustrate how the effective frequency span of the transverse impedance affects the predicted transverse stability with the lefthand panel of Fig. 22. Here, we show that at zero chromaticity the predicted instability threshold is relatively insensitive to the wakefield solver bunch length $\bar{\sigma}_{s}$, with $\bar{\sigma}_{s}$ only playing a role when it approaches the electron bunch length itself. This is because at $\xi=0$, the instability is largely rigid in the longitudinal plane, and dominantly driven by the impedance near zero frequency. In contrast to this, at nonzero chromaticity the relevant frequency is shifted by the chromatic frequency shift $\omega_{\xi}$, head-tail dynamics results in important longitudinal structure, and the impedance range needs to include these effects. In Fig. 22, we find that the instability threshold at large chromaticity can be significantly underestimated if the wakefield resolution $\bar{\sigma}_{s}$ is not sufficient. 

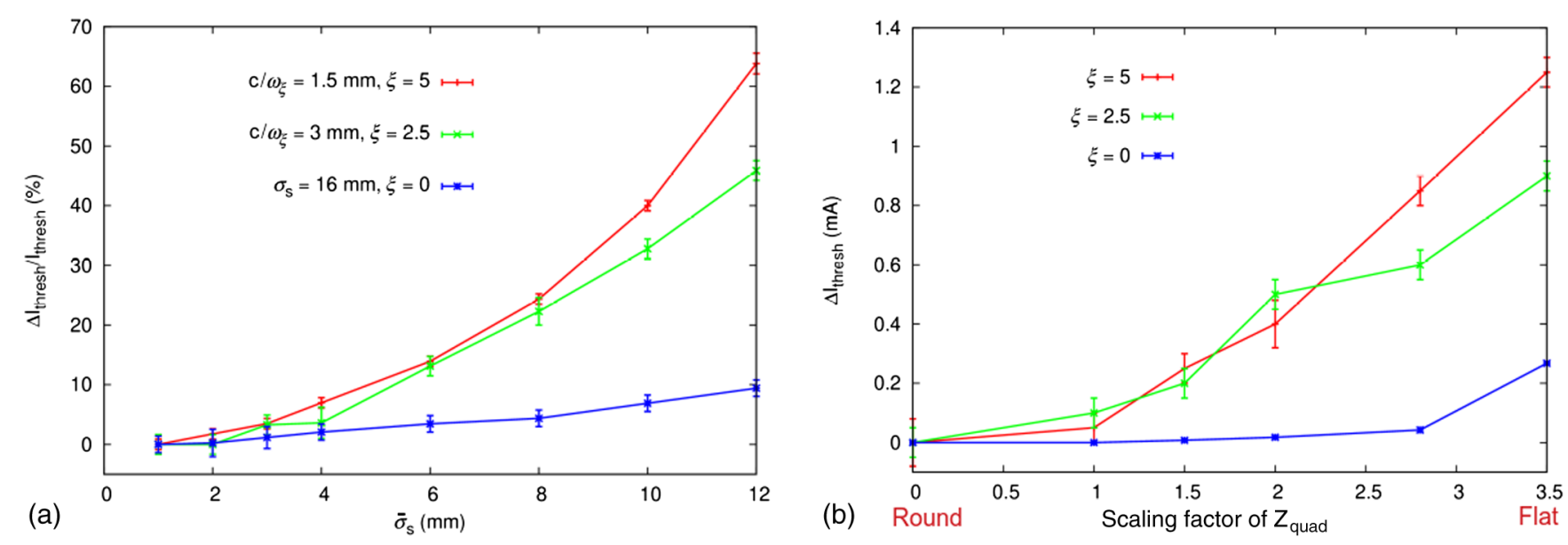

FIG. 22. Dependence of the predicted transverse instability threshold as a function of the transverse impedance extent as characterized by the wakefield solver bunch length $\bar{\sigma}_{s}$. Predicted instability threshold as a function of the strength of the quadrupolar wakefield for the APS-U, which has most round chambers.

Finally, the simulations should include both the dipolar wakefield (in which the kick is proportional to the displacement of the source particle) and quadrupolar wakefield (where the kick scales with the displacement of the test particle). Note that this is true even though only the dipole wakefield directly drives the instability, since the quadrupolar wakefield results in a tune shift that can indirectly affect stability [91]. We illustrate this in the right-hand panel of Fig. 22, where we have (somewhat artificially) scaled the predicted APS-U quadrupolar impedance while keeping the dipole impedance fixed. The scaling factor 1 corresponds to that predicted for the APS-U, while setting the quadrupolar impedance to zero assumes round chambers everywhere. Since the APS-U has mostly round chambers the limit of entirely flat chambers leads to increasing the quadrupolar impedance by a factor of 3.5 . Figure 22 shows that these variations can lead to significantly different conclusions regarding transverse stability. Although this example is somewhat contrived, it does illustrate the fact that the quadrupolar impedance can play an important role in predicting transverse stability.

\section{CONCLUSIONS}

In this paper, we discussed the methods we have used to quantify collective effects in a storage ring. These methods were used during the design and operating phases of the accelerator facility, and involved close coordination of many groups including those covering accelerator physics, vacuum design, diagnostics, rf, and IDs. A well-estimated impedance budget is an important part of predicting the performance of facilities with intense beams, and such predictions should be based on vacuum component designs as installed in the ring. This is particularly important during the final stages of component design, manufacture, and installation, when seemingly small changes can be introduced that may alter the associated impedance.
Once the impedance has been computed, we have shown how to include collective effects in simulations, paying particular attention to practical considerations that can affect the simulations fidelity. We have indicated that accurate modeling of the longitudinal dynamics is a prerequisite for obtaining reliable transverse calculations, and indicated how we have developed consistent tracking results. In addition to instability calculations, we have discussed $\mathrm{rf}$ heating as another issue that may limit beam intensity, and discussed methods to predict such heating. We hope that such descriptions will prove useful to other groups who want to understand collective behavior as part of accelerator and ring design.

\section{ACKNOWLEDGMENTS}

This work was supported by Brookhaven Science Associates, LLC under Contract No. DE-SC0012704 with the U.S. Department of Energy. The work by R. Lindberg was supported by Department of Energy Contract No. DEAC02-06CH11357. We would like to thank G. Wang (RHIC) for his help with the ECHO 3D simulation for the NSLS-II flange absorber and K. Shibata (SKEKB) and H. O. Duarte (Sirius) for providing the geometric dimensions of the comb-type bellows and cross-checking the numerically simulated results. We thank C. Hetzel (EIC) for his numerous vacuum system related support. Finally, we would like to acknowledge M. Borland for help with elegant and a fruitful discussion, L. Emery, Y.-C. Chae, and V. Sajaev for sharing APS data.

[1] P. Den Hartog, J. Gagliano, G. Goeppner, J. Noonan, E. Trakhtenberg, and G. Wiemerslage, Vacuum system of the APS: Operation experience and status report, in Proceed- 
ings of the 2001 Particle Accelerator Conference, Chicago, 2001 (JACoW, Geneva, 2001).

[2] Brookhaven National Laboratory, NSLS-II Conceptual Design Report, 2006, https://www.bnl.gov/nsls2/project/ $\mathrm{CDR} /$.

[3] R. Nagaoka, Fast beam-ion instability arising from local outgassing, in Proceedings of the Topical Workshop on Instabilities, Impedance and Collective Effects 2014 (TWIICE 2014) (Synchrotron SOLEIL, 2014).

[4] R. P. Walker, Commissioning and status of the DIAMOND storage ring, Raja Ramanna Centre for Advanced Technology(RRCAT), Indore, India, TUYMA03, Technical Report No. APAC 2007.

[5] MAX-IV Detailed Design Report, available at http://www .maxiv.lu.se/publications.

[6] C. Steier et al., Status of the conceptual design of ALS-U, in Proceedings of IPAC2017, WEPAB104, Copenhagen, Denmark, 2017.

[7] J. Carter, B. Billett, B. Brajuskovic, M. Lale, A. McElderry, J. Noonan, M. O'Neill, K. Wakefield, D. Walters, G. Wiemerslage, and J. Zientek, in MEDSI2018 Proceedings of the 10th Mechanical Engineering Design of Synchrotron Radiation Equipment and Instrumentation (JACoW, Geneva, 2018), https://doi.org/10.18429/JACoWMEDSI2018-TUPH04.

[8] R. M. Seraphim, O. R. Bagnato, R. O. Ferraz, H. G. Filho, G. R. Gomes, M. Nardin, R. F. Oliveira, B. M. Ramos, T. M. Rocha, A. R. D. Rodrigues, and M. B. Silva, Vacuum system design for the Sirius storage ring, in Proceedings of IPAC2015, WEPMA003, Richmond, VA, USA, 2015 (JACoW, Geneva, 2015).

[9] PETRA-IV Conceptual Design Report, https://bib-pubdb1 .desy.de/record/426140/files/DESY-PETRAIV-

Conceptual-Design-Report.pdf.

[10] SLS-2 Conceptual Design Report, 2017, https://www.dora .lib4ri.ch/psi/islandora/object/psi\%3A34977/datastream/ PDF/Streun-2017-SLS-2._Conceptual_design_report-\% 28published_version\%29.pdf.

[11] EBS Storage Ring Technical Report, 2018, https://www .esrf.fr/files/live/sites/www/files/about/upgrade/ documentation/Design\%20Report-reduced-jan19.pdf.

[12] M. P. Cox, C. W. Burrows, A. G. Day, J. A. DymokeBradshaw, R. K. Grant, N. P. Hammond, X. Liu, A. G. Miller, H. S. Shiers, and N. R. Warner, Conceptual design of the DIAMOND-II vacuum system, in Proceedings of IPAC2019, TUPMP050, Melbourne, Australia, 2019 (JACoW, Geneva, 2019).

[13] D. Einfeld et al., Design of a diffraction limited light source (DIFL), in Proceedings of PAC'95, Dallas, 1995 (JACoW, Geneva, 1995), p. 177.

[14] M. Borland, T. Berenc, R. Lindberg, and A. Xiao, Tracking studies of a higher-harmonic bunch-lengthening cavity for the APS Upgrade, in Proceedings of IPAC2015, MOPMA007, Richmond, VA, USA, 2015 (JACoW, Geneva, 2015).

[15] R. Lindberg, Theory of coupled-bunch longitudinal instabilities in a storage ring for arbitrary RF potentials, Phys. Rev. Accel. Beams 21, 124402 (2018).

[16] R. Warnock and M. Venturini, Equilibrium of an arbitrary bunch train in presence of a passive harmonic cavity:
Solution through coupled Haissinski equations, arXiv: 2003.09959.

[17] M. Venturini, Passive higher-harmonic RF cavities with general settings and multibunch instabilities in electron storage rings, Phys. Rev. Accel. Beams 21, 114404 (2018).

[18] N. Towne and J. Rose, Bunch lengthening harmonic system for NSLS-II, in Proceedings of EPAC08, Genoa, Italy, 2008 (JACoW, Geneva, 2008), paper MOPP152.

[19] M. Georgsson, A. Andersson, and M. Eriksson, Landau cavities at MAX-II, Nucl. Instrum. Methods Phys. Res., Sect. A 416, 465 (1998).

[20] J. M. Byrd, S. De Santis, M. Georgsson, G. Stover, J. D. Fox, and D. Teytelman, Commissioning of a higher harmonic RF system for the Advanced Light Source, Nucl. Instrum. Methods Phys. Res., Sect. A 455, 271 (2000).

[21] M. Pedrozzi et al., SLS operational performance with third harmonic superconducting system, in Proceedings of 11th Workshop on RF superconductivity, Lubeck, Germany.

[22] M. Svandrlik et al., Performance of the 3rd harmonic superconducting cavity at ELLETRA, in Proceedings of 11th Workshop on RF superconductivity, Lubeck, Germany.

[23] W. Anders and P. Kuske, HOM damped NC passive harmonic cavities at BESSY, in Proceedings of 2003 Particle Accelerator Conference (JACoW, Geneva, 2003), p. 1186.

[24] D. Alesini et al., The DAFNE 3rd harmonic cavity, in Proceedings of 2001 Particle Accelerator Conference, Chicago (JACoW, Geneva, 2001), p. 885.

[25] A. Blednykh et al., Harmonic cavity performance for NSLS-II, in Proceedings of 2005 Particle Accelerator Conference, Knoxville, Tennessee, p. 2544 (JACoW, Geneva, 2005).

[26] G. Bassi and Juri Tagger, Longitudinal beam dynamics with a higher harmonic cavity for bunch lengthening, Int. J. Mod. Phys. A 34, 1942040 (2019).

[27] G. Skripka et al., Commissioning of the harmonic cavities in the MAX IV $3 \mathrm{GeV}$ storagering, in Proceedings of IPAC2016, Busan, Korea, 2016 (JACoW, Geneva, 2016), WEPOW035.

[28] H. Feng et al., Design study on higher harmonic cavity for ALS-U, in Proceedings of IPAC2019, Melbourne, Australia, 2019 (JACoW, Geneva, 2019), WEPRB081.

[29] W. Bruns, http://www.gdfidl.de.

[30] CST Particle Studio, http://www.3ds.com.

[31] ANSYS HFSS, https://www.ansys.com.

[32] I. Zagorodnov and T. Weiland, TE/TM field solver for particle beam simulations without numerical Cherenkov radiation, Phys. Rev. ST Accel. Beams 8, 042001 (2005).

[33] I. Zagorodnov, Indirect methods for wake potential integration, Phys. Rev. ST Accel. Beams 9, 102002 (2006).

[34] I. Zagorodnov, https://echo4d.de/.

[35] U. Laustroer, U van Rienen, and T. Weiland, URMEL and URMEL-T user guide, DESY, Report No. M-87-03, 1987.

[36] VORPAL, https://ice.txcorp.com.

[37] ACE3P, https://conf.slac.stanford.edu/cw18/sites/cw18.conf .slac.stanford.edu/files/broc-ace3p.pdf.

[38] Yong Ho Chin, http://abci.kek.jp/abci.htm.

[39] SuperFish, https://laacg.lanl.gov/laacg/services/.

[40] H. O. C. Duarte, Impedance optimization and wakefield codes comparison for the Brazilian Light Laboratory, in 
Proceedings of Topical Workshop on Instabilities, Impedance and Collective Effects 2014, TWIICE2014.

[41] V. Smaluk, R. Fielder, A. Blednykh, G. Rehm, and R. Bartolini, Coupling impedance of an in-vacuum undulator: Measurement, simulation, and analytical estimation, Phys. Rev. ST Accel. Beams 17, 074402 (2014).

[42] Handbook of Accelerator Physics and Engineering, edited by A.W. Chao and Maury Tigner (World Scientific Publishing Co., Singapore, 2013), 2nd ed., Chap. 3.

[43] L.-Q. Lee, A. Candel, C. Ng, and K. Ko, A moving window technique in parallel finite element time domain electromagnetic simulation, Technical Report No. SLACPUB-14099, 2010.

[44] I. Zagorodnov, Wakefield code ECHO, algorithms and applications, in Proceedings of workshop on Instabilities, Impedance and Collective Effects, Abingdon, Oxfordshire, 2016.

[45] K. Oide and K. Yokoya, Longitudinal single-bunch Instability in electron storage ring, KEK Preprint 90-10, 1990.

[46] D. Boussard, Observation of microwave longitudinal instabilities in the CPS, CERN, Technical Report no. LabII/ RF/INT/75-2, 1975.

[47] R. Lindberg and A. Blednykh, Instability thresholds for the advanced photon source multi-bend achromat upgrade, in Proceedings of the 6th International Particle Accelerator Conference IPAC2015, TUPJE077, Richmond, VA, USA, 2015 (JACoW, Geneva, 2015).

[48] A. Blednykh, G. Bassi, M. Blaskiewicz, C. Hetzel, V. Ptitsyn, V. Smalyuk, and F. Willeke, Impedance modeling for eRHIC, in Proceedings of the 9th International Particle Accelerator Conference IPAC2018, TUPMF027, Vancouver, BC, Canada, 2018 (JACoW, Geneva, 2018).

[49] A. Piwinsky, Wakefields and Ohmic losses in flat vacuum chambers, Deutsches Elektronen-Synchrotron DESY, Technical Report No. DESY-HERA-92-04, 1992.

[50] K. Bane and M. Sands, The short-range resistive wall wakefields, Stanford University, Technical Report No. SLAC-PUB-95-7074, 1995.

[51] K. Yokoya, Resistive wall impedance of beam pipes of general cross section, Part. Accel. 41, 221 (1993).

[52] A. Piwinsky, Wakefields and Ohmic losses in round vacuum chambers, Deutsches Elektronen-Synchrotron DESY, Technical Report No. DESY-HERA-92-11, 1992.

[53] R. Bartolini (private communication).

[54] T. Ha (private communication).

[55] A. Blednykh, B. Bacha, G. Bassi, G. Ganetis, C. Hetzel, H.-C. Hseuh, T. Shaftan, V. Smaluk, and G. Wang, Beaminduced heating of the kicker ceramics chambers at NSLS-II, in Proceedings of NAPAC2016, Chicago, IL, USA, 2016 (JACoW, Geneva, 2016).

[56] A. Piwinski, Penetration of the field of a bunched beam through a ceramic vacuum chamber with metallic coating, IEEE Trans. Nucl. Sci. 24, 3 (1977).

[57] ImpedanceWake2D, https://twiki.cern.ch/bin/view/ ABPComputing/ImpedanceWake2D.

[58] B. W. Zotter and S. A. Kheifets, Impedance and Wakes in High-Energy Particle Accelerators (World Scientific, Singapore, 1997).

[59] A. Burov and V. Lebedev, Transverse resistive wall impedance for multi-layer round chambers, in Proceedings of EPAC 2002 (JACoW, Geneva, 2002), pp. 1452-1455.
[60] E. Koukovini-Platia, G. Rumolo, and C. Zannini, Electromagnetic characterization of nonevaporable getter properties between $220-330$ and $500-750 \mathrm{GHz}$ for the Compact Linear Collider damping rings, Phys. Rev. Accel. Beams 20, 011002 (2017).

[61] G. Stupakov, Low frequency impedance of tapered transitions with arbitrary cross sections, Phys. Rev. ST Accel. Beams 10, 094401 (2007).

[62] R. L. Gluckstern, Coupling impedance of a single hole in a thick-wall beam pipe, Phys. Rev. A 46, 1106 (1992).

[63] J. J. Bisognano, S. A. Heifets, and B. C. Yunn, Loss parameters for very short bunches, in Proceedings of EPAC 1988, Rome, Italy, 1988 (JACoW, Geneva, 1988), Vol. 88, p. 708.

[64] G. Stupakov, K. L. F. Bane, and I. Zagorodnov, Impedance scaling for small angle transitions, Phys. Rev. ST Accel. Beams 14, 014402 (2011).

[65] G. Stupakov, K. L. F. Bane, and I. Zagorodnov, Optical approximation in the theory of geometric impedance, Phys. Rev. ST Accel. Beams 10, 054401 (2007).

[66] A. Blednykh, B. Bacha, G. Bassi, C. Hetzel, B. Kosciuk, D. Padrazo, T. Shaftan, V. Smaluk, and T. Ha, Beam impedance and heating analysis of the diagnostic stripline, Nucl. Instrum. Methods Phys. Res., Sect. A 963, 163729 (2020).

[67] G. R. Lambertson, Dynamic devices-pickups and kickers, in Physics of Accelerators, edited by M. Month and M. Dienes [AIP Conf. Proc. 153, 1413 (1987)].

[68] K.-Y. Ng, Impedances of stripline beam-position monitors, Part. Accel. 23, 93 (1988).

[69] A. Blednykh, G. Bassi, C. Hetzel, B. Kosciuk, D. Padrazo, V. Smaluk, T. Shaftan, and G. Wang, Temperature measurements of the NSLS-II vacuum components, in Proceedings of North American Particle Accelerator Conference, NAPAC2019, TUPLM36, Lansing, Michigan, 2019 (JACoW, Geneva, 2019).

[70] V. Kiselev and V. Smalyuk, Experimental study of impedances and instabilities at the VEPP-4M storage ring, in Proceedings of the 1999 DIPAC, Chester, United Kingdom, 1999 (JACoW, Geneva, 1999), pp. 1005.

[71] Emery, G. Decker, and J. Galayda, Local bump method for measurement of transverse impedance of narrow-gap ID chambers in storage rings, in Proceedings of the 2001 PAC, Chicago, USA, 2001 (JACoW, Geneva, 2001), pp. 1823.

[72] S.S. Kurennoy, Beam coupling impedances of obstacles protruding into a beam pipe, Phys. Rev. E 55, 3529 (1997).

[73] S. S. Kurennoy and R. L. Gluckstern, Impedances of azimuthally symmetric irises and cavities with semielliptical profile in a beam pipe, Phys. Rev. E 55, 3533 (1997).

[74] G. V. Stupakov, Impedance of small obstacles and rough surfaces, Phys. Rev. ST Accel. Beams 1, 064401 (1998).

[75] Brookhaven National Laboratory, EIC Conceptual Design Report 2021, https://www.bnl.gov/ec/files/EIC_ CDR_Final.pdf.

[76] A. Blednykh, M. Blaskiewicz, D. Gassner, C. Hetzel, B. Lepore, B. Podobedov, V. Ranjbar, M. Sangroula, P. Thieberger, S. Verdu-Andres, G. Wang, and Q. Wu, An overview of the collective effects and impedance calculation for the EIC, in Proceedings ofIPAC2021, THPAB238, Campinas, SP, Brazil, 2021 (JACoW, Geneva, 2021). 
[77] Y. Suetsugu, M. Shirai, and K. Shibata, Possibility of comb-type RF shield structure for high-current accelerators, Phys. Rev. ST Accel. Beams 6, 103201 (2003).

[78] Y. Suetsugu, K. Kanazawa, N. Ohuchi, K. Shibata, and M. Shirai, Application of comb-type RF shield to bellows chambers and gate valves, in Proceedings of 2005 Particle Accelerator Conference, Knoxville, Tennessee, 2005 (JACoW, Geneva, 2005).

[79] H. O. C. Duarte, R. M. Seraphim, T. M. Rocha, A. R. D. Rodrigues, and P. P. S. Freitas, Design review of bellows RF-shielding types and new concepts for Sirius, in Proceedings of IPAC2019, Melbourne, Australia, doi: https:// doi.org/10.18429/JACoW-IPAC2019-MOPGW001.

[80] K. Yokoya, Impedance of slowly tapered structures, CERN, Geneva, Technical Report No. CERN-SL-90-88AP, 1990.

[81] G. V. Stupakov, Geometrical wake of a smooth taper, Part. Accel. 56, 83 (1996).

[82] A. Blednykh, G. Bassi, C. Hetzel, B. Kosciuk, and V. Smaluk, NSLS-II longitudinal impedance budget, Nucl. Instrum. Methods Phys. Res., Sect. A 1005, 165349 (2021).

[83] V. Smaluk, Impedance computations and beam-based measurements: A problem of discrepancy, Nucl. Instrum. Methods Phys. Res., Sect. A 888, 22 (2018).
[84] A. W. Chao, Physics of Collective Beam Instabilities in High Energy Accelerators (Wiley, New York, 1993).

[85] ANSYS Academic Research Mechanical, Release 18.1.

[86] A. Blednykh, B. Bacha, G. Bassi, W. Cheng, O. Chubar, A. Derbenev, R. Lindberg, M. Rakitin, V. Smaluk, M. Zhernenkov, Yu-Chen Karen Chen-Wiegart, and L. Wiegart, New aspects of longitudinal instabilities in electron storage rings, Sci. Rep. 8, 11918 (2018).

[87] V. Sajaev, R. Lindberg, M. Borland, and S. Shin, Simulations and measurements of the impact of collective effects on dynamic aperture, Phys. Rev. Accel. Beams 22, 032802 (2019).

[88] B. Podobedov and G. Stupakov, Point-charge wakefield calculations from finite length bunch wake potentials, Phys. Rev. ST Accel. Beams 16, 024401 (2013).

[89] R. Nagaoka, Instability studies using evaluated wake fields and comparison with observations at SOLEIL, Proc. EPAC 2006, 2847 (2006).

[90] B. Zotter and F. Sacherer, Instabilities of relativistic particle beams in accelerators and storage rings, in Proceedings of First Course of International School of Particle Accelerator of the 'Ettore Majorana' (JACoW, Geneva, 1976), CERN 77-13, p. 175.

[91] A. Burov and V. Danilov, Suppression of Transverse Bunch Instabilities by Asymmetries in the Chamber Geometry, Phys. Rev. Lett. 82, 2286 (1999). 